\title{
A theory of causation: causae causantes (Originating causes) as inus conditions in branching space-times
}

\author{
Nuel Belnap
}

November 19,2002

\begin{abstract}
Branching space-times (BST) theory, as developed elsewhere, permits a sound and rigorously definable notion of "originating cause" or causa causans - a type of transition event — of an outcome event. Mackie has famously suggested that causes form a family of "inus" conditions, where an inus condition is "an insufficient but non-redundant part of an unnecessary but sufficient condition." In this essay the needed concepts of BST theory are developed in detail, and it is then proved that the causae causantes of a given outcome event have exactly the structure of a set of Mackie inus conditions. The proof requires the assumption that there is no EPR-like "funny business." This seems enough to constitute a theory of "causation" in at least one of its many senses.
\end{abstract}

\section{Introduction}

One notion of causation comes from the practice of accounting for a certain outcome event by tracing back to the events that stand as its originating causes, skipping over any intermediate deterministic processes. One is asking for the "choice points" such that what happens there makes a difference to whether the given outcome event occurs. I aim to elucidate such a notion by finding that it is already present in a certain rigorous axiomatic theory, the theory of branching space-times.

Mackie 1974 says that an objective and directional concept of causation requires objective indeterminism. Indeterminism is well represented by the muchstudied tree-like structures of "branching time," and both von Kutschera 1993 and Xu 1997 develop theories of causation in branching time. These articles are foundational explorations of how indeterminism is relevant or even essential for causal- 
ity. The need for indeterminism may be put as follows: Given universal determinism, there is no room for the idea of really possible alternatives (Xu speaks of "possibility based on reality"). Von Kutschera and Xu both provide telling arguments in favor of the relevance of indeterminism to causation.

The aim of the present essay is to explore the use of "branching space-times" (BST) theory as a suitable framework in which further to develop ideas of causation requiring indeterminism. The advantage of BST as over against branching time is that only the former can represent causes and effects as local rather than as worldwide.

BST theory takes as its chief primitive a "causal ordering" relation $<$ among the concrete possible point events of Our World. BST in the form studied here comes from Belnap 1992, and has been elaborated in Belnap 2002a and Belnap 2002b; I will occasionally refer to these three articles with the phrase "earlier BST essays."1 BST is a common generalization of a relativistic Minkowski-type space-time on the one hand, and of indeterminist branching time on the other: BST is both relativistic and indeterminist.

A theory of "causal order" is not a theory of causality, an observation made obvious by considering the causal order of either Minkowski space-time or branching time. No one thinks that if the causal-order relation $<$ holds between two events, so that the first is in the "causal past" of the second, then the earlier event "is a cause of" the later event! Nevertheless, there are stories to be told of causality in BST. This one is about tracing causality back to its beginnings in objectively indeterministic originating causes or causae causantes (I use these as synonyms). In this respect it is indebted to the account of agency of Belnap, Perloff and Xu 2001, which in outline is the same as the much earlier theory of von Kutschera 1986. The "payoff" will be a technical result in the BST theory of indeterministic causal structure the point of which comes from a certain idea of Mackie 1974 on causation, namely, the idea of an inus condition:

1-1 QUASI-DEFINITION. (Inus condition) An inus condition of an event type is "an insufficient but non-redundant part of an unnecessary but sufficient condition" (p. 62).

The surrounding text makes it clear that Mackie has in mind a disjunction of conjunctions such as " $A B C$ or $D G H$ or $J K L$ " (this is his example) such that the whole disjunction is a necessary condition of (say) $P$ (this feature is implicit in Mackie's formula), and each disjunct is sufficient for $P$, and if any element such

\footnotetext{
${ }^{1}$ These earlier BST essays contain extensive motivation as well as technical development that cannot usefully be repeated here. For closely related alternative versions of branching space-times, see Müller 2002 and Placek 2002b and the works cited therein.
} 
as $A$ is omitted from its conjunction, then the remaining conjuncts are no longer sufficient for $P$. Under these circumstances, $A$ is called an "inus condition" of $P$. In an alternate mouthful, $A$ is a non-redundant conjunctive part of a sufficient condition for $P$ that is a disjunctive part of a necessary condition for $P$.

The central-and perhaps surprising — result of this essay is that the causae causantes of BST have exactly this structure.

\section{The cement of the universe}

I indicate some of the complex ways that the BST account of causae causantes as inus conditions relates to Mackie's ideas.

"I think we can and sometimes do think and speak of such concrete occurrences as causes" (p. 257), and Mackie distinguishes on p. 265 between "explaining causes" (facts) and "producing causes" (events). Given this dichotomy, I shall here be concerned only with producing causes. In exactly the same way, BST theory concerns itself with produced effects or results.

In spite of allowing events as causes, for Mackie inus conditions are types of events, types that may have instances (p. 62). In the present development, however, inus conditions are concrete possible events (in one of the several senses of "event" countenanced by BST theory); they are neither types nor instances of types (except of course in the sense in which everything is an instance of arbitrarily many types).

BST theory has very little to say about explanation. It is not, however, accurate to say that BST does not at all concern itself with "facts." Indeed, it is a virtue of BST theory that it provides clear and rigorous and decidedly nontrivial links between local concrete events and certain "facts," namely, propositions to the effect that a certain event "occurs."

Let it be noted that "occurs" makes no sense for an event in the sense of a hunk of space-time. Mackie recognizes this: "Causation is not something between events in a spatio-temporal sense ...." In a crucially important shift, BST theory looks for causation as a relation between possible events, where it is understood that events are concrete possibilia. This relation has a spatio-temporal component, so to speak, as well as a modal component.

Although taking careful note of Vendler 1962, Mackie tends to assimilate causes and effects with regard to ontological category: Either can be a fact, either can be an event, but the point is that whichever can be one can also be the other. The present theory comes out, after analysis, with a similar view, driven, however, not by "ordinary language," but by considerations that BST theory suggests to be in the nature of the case. As a first approximation, what is caused is said to involve an event that can begin; the BST jargon is that the caused involves an outcome event. 
After refinement, the caused is described as a transition from an initial event to an outcome event. The causing inus condition is also taken to be a transition, but of a very special kind: The suggestion is that an inus condition should be identified as a causa causans, which in turn is described as a species of immediate contingent transition, with no room between initial and outcome. It is an additional point that at the same time, we shall need to keep track of both the "event" and the "fact" aspects of both causing and caused, noting that their roles are by no means interchangeable. Quite generally, in BST it is natural to articulate a typology of entities useful in talking of causation, and a companion family of predicates for characterizing entities of various types. The primitive ontology of BST is meager: Our World is a set of point events structured by a simple causal-order relation. There are, however, an array of "derived" (definable) types of entities, and it is these that prove useful in thinking about causation.

Mackie is concerned with a concept of "causation in the objects," in contrast to psychological or linguistic or even epistemic notions. BST theory is on the same side as Mackie with respect to those contrasts, but with an additional contrast as well. Instead of objects, BST looks for a concept of "causation in the events." This records an explicit limitation of BST theory: Mackie has something to say about persistence, continuity, regularity, whereas the language of BST, concerning as it does only the causal structure of events, is expressively inadequate to these important ideas. (BST is not a theory of everything.)

In Mackie's view the "direction of causation" requires objective indeterminism (p. 191), something that is also an absolute requirement of BST theory.

Finally, Mackie's theory permits the possibility of "backward causation." Various analytical shifts make it difficult to compare Mackie on this point directly with BST theory, but the following is true and may help. Central to BST theory, as explained in $\S 4.2$, is the notion of a "cause-like locus" for an outcome event. It follows from the governing postulates of BST theory that no cause-like locus for an outcome event can lie in the future of the event, which is perhaps a difference from Mackie. BST theory, however, leaves open whether or not every cause-like locus must occur in the past; some such loci might, as far as BST theory goes, be space-like related to the outcome event, and so neither past nor future. This seems to happen in strange EPR-like quantum-mechanical cases, cases of what I call "funny business." In framing an inus theory of causation, however, I make the further assumption that there is no funny business, so that every cause-like locus for a certain outcome event definitely lies in the past of that event. Hence, the present story about inus conditions is not known to work in the presence of quantum-mechanical funny business. 


\section{Preliminaries}

An essential feature of this account is that there is no arm-waving, a feature, shared by the accounts of causation based on branching time mentioned in $\S 1$ and the alternative accounts of branching space-times to which I referred in note 1 . The reader will find BST laid out as an exact theory in the earlier BST essays, to which I must refer for a systematic presentation of notation, postulates and definitions. In this section I go over what is needed for a theory of causation.

\subsection{First definitions and postulates}

The primitives of BST are two: Our World, a set of "concrete possible point events" (or just "point events") $e$, and $<$, the "causal order" on Our World. The causalordering relation $e_{1}<e_{2}$, which has both spatio-temporal and modal significance in BST, may be read as either " $e_{1}$ is in the settled causal past of $e_{2}$ " or " $e_{2}$ is in the future of possibilities of $e_{1}$." It is assumed that $<$ is a dense strict partial order on Our World with no maximal elements. I let $h$ be a history, i.e., a maximal directed set, where a set is directed if it contains an upper bound for each pair of its members. Fact: Histories are closed downward. Hist is the set of all histories of Our World. $H$ is a set of histories (also called a proposition). $-H={ }_{d f} H i s t-H$.

There are a (very) few other postulates; of these far and away the most important is the "prior choice postulate." I give only its bare statement here. Let $O$ be a nonempty and lower-bounded chain of point events (D5 below calls $O$ an "outcome chain"), and let $O \subseteq h_{1}-h_{2}$. Then there is a point event $e$ in the causal past of every member of $O$ such that $e$ is maximal in $h_{1} \cap h_{2}$.

\subsection{Ontology: propositions}

The idea of inus conditions (Quasi-definition 1-1) requires (at least) the language of sufficient and necessary conditions, which is part of the language of propositions. $\S 3.1$ defined propositions as sets of (not worlds but) histories in Our World. This section spells out, in strict analogy to possible-worlds theory, the BST version of key parts of the language of propositions. BST theory, however, goes beyond possible-worlds theory when it indicates how propositional language can be intelligently and exactly applied to concrete events. I begin this transference of propositional language to events in this section, but it is only completed in $\S 3.6$.

\section{The language of propositions.}

D1. A proposition $H$ is defined as true or false in a history according as to whether or not $h \in H$. 
$H$ is consistent $\leftrightarrow_{d f} H \neq \varnothing . \mathbf{H}$ is a set of sets of histories (hence a set of propositions); $\mathbf{H}$ is jointly consistent $\leftrightarrow_{d f} \bigcap \mathbf{H} \neq \varnothing . H$ is universal $\leftrightarrow_{d f}$ $H=H i s t$, and $H$ is contingent $\leftrightarrow_{d f} H$ is consistent but not universal. $H_{1}$ implies $H_{2} \leftrightarrow_{d f} H_{1} \subseteq H_{2}$; we may also say that $H_{1}$ is a sufficient condition for $\mathrm{H}_{2}$, or that $\mathrm{H}_{2}$ is a necessary condition for $H_{1}$.

Sometimes I include a modifier "historically" (for example, "historically necessary condition") to make it explicit that these properties and relations are not "logical" and do not involve reference to "possible worlds."

Propositional language applied to point events. I shall be mentioning a number of types of events, specifying as they are introduced exactly what it means to say of an event of a certain type that it occurs. On the BST account, events occur (or not) in a history; that is how the occurrence of an event can be contingent. The order of definition shall be this. First, for an event of a certain type, I define its occurrence proposition. Then the following usages are uniform.

D2. An event occurs in a history $h$ iff its occurrence proposition is true in $h$, which, by D1, holds iff $h$ is a member of its occurrence proposition.

That is how BST theory makes room for both events and "facts." I I begin here with an application of propositional language to point events.

D3. $H_{(e)}={ }_{d f}\{h: e \in h\}$ is the occurrence proposition for $e$, true in $h$ iff $h \in$ $H_{(e)}$.

In this case as in others to come, given an event, there is in BST theory a unique matching occurrence proposition; but you should not expect uniqueness in the other direction. BST does not forbid, for instance, that distinct point events $e_{1}$ and $e_{2}$ should occur in exactly the same histories: $H_{\left(e_{1}\right)}=H_{\left(e_{2}\right)}$, but $e_{1} \neq e_{2}$. In the customary language of events and facts, we may say that specification of an event usually gives more information than specification of an occurrence-fact.

\footnotetext{
${ }^{2}$ Belnap et al. 2001, especially chapter 6, argues at length that if one wishes to be clear about indeterminism, actuality (or factuality) needs to be taken indexically as whatever is settled true in the context of utterance (see Definition 7-1(6) below). It is part of the story that the idea of a single "actual history" is a bad idea, even if taken indexically. There is no need to repeat those arguments here, but the conclusion should be borne in mind. In particular, when I say "fact" in deference to customary usage, I usually mean just "proposition," but if I have gone to the trouble of indicating a point event to be taken as an idealized context of utterance, you will do no wrong if you identify "fact" with "proposition that is settled true-in the sense of Definition 7-1(4)—at the context of utterance."
} 


\subsection{Ontology: initial events}

In order to understand causation, we must be able to think about events, both causeevents and effect-events, that are essentially transition events. ${ }^{3}$ If lighting the match is causally related to there being a fire, we shall need to think about two transition events: The lighting as cause is a transition from match-not-lit to match-lit, and the fire as effect is a transition from no-fire to fire. Von Wright 1963 labeled his generic state-to-state transitions events (p. 27), and while BST does not deal in states, the fundamental idea is the same: BST imitates Russell's account of motion by offering an "at-at" theory of transition events. ${ }^{4}$ That is, we shall identify a transition event as nothing more than (nor less than) an ordered pair consisting of an initial event and an outcome event. I systematically use "_ $\longrightarrow$ " for every transition event, relying on the reader to appreciate that the arrow is not supposed to represent some kind of mysterious glue "between" events: _ ${ }_{-}$is just an ordered pair.

It is a conceptual feature of BST theory that a transition event, as defined in $\S 3.5$, has both an initial and an outcome. Each of these, in turn, is an event of a certain kind; there is no avoiding this complexity. First I introduce initial events. ${ }^{5}$

D4. $\mathbf{I}$ is an initial event $\leftrightarrow_{d f} \mathbf{I}$ is a nonempty set of point events all of which are members of some one history. An initial event may be scattered both space-like and time-like.

$H_{[\mathbf{I}]}={ }_{d f}\{h: \mathbf{I} \subseteq h\}$ is the occurrence proposition for $\mathbf{I}$, true in $h$ iff $h \in H_{[\mathbf{I}]}$.

Typically an initial event $\mathbf{I}$ occurs in many histories, but those histories do not split indeterministically until after $\mathbf{I}$. Place yourself inside $\mathbf{I}$ at a particular point event $e \in \mathbf{I}$. From that vantage point, it is a settled matter that earlier portions of $\mathbf{I}$ occur, but inside $\mathbf{I}$ it is typically not settled that $\mathbf{I}$ occurs (it ain't over 'till it's over). If, however, a point event $e$ is in the future of all of $\mathbf{I}$, then at $e$ it is a settled matter that $\mathbf{I}$ occurs (when it's over it's over). For an initial to occur in a history, all of the initial must be part of the history. If you set up a measurement on the $x$ axis, that set-up event does not occur in a history unless it is complete; that is the way of initials. When we set up an experiment, we don't look for an outcome or result of that particular set up until all parts of the setting up have run to completion. Also keep in mind that initials are designed to be initials of transition events, and consider how essential it is - at least in clear cases - for the initial of a transition to be complete before the outcome commences.

\footnotetext{
${ }^{3}$ Transition events are fundamental to Xu's account of causation, which should be consulted.

${ }^{4}$ Belnap 1999 contains an extended informal discussion of concrete transitions.

5"Initial chains" I (nonempty and upper bounded chains), were needed in earlier BST essays, but they do not play a role in this study.
} 
A single point event $e$ also counts as an initial event, thus "identifying" the point event $e$ with the "official" initial event $\{e\}$. Accordingly, transition events are going to have one of the two forms $\mathbf{I} \succ \ldots$ and $e \longmapsto \ldots$, with the second being really a special case of the first.

\subsection{Ontology: outcome events}

I now turn to kinds of "outcome events" needed to fill the second position of a transition event _ $\longmapsto$. It turns out that we must consider three kinds of "outcome events" of increasing complexity, each with its own occurrence proposition.

Outcome chains. The simplest type of outcome event is the outcome chain.

D5. $O$ is an outcome chain $\leftrightarrow_{d f} O$ is a nonempty and lower bounded chain, where a set is a chain if each two of its members are comparable by $<$. Provably $O$ $\subseteq h$ for some $h$.

$H_{\langle O\rangle}=_{d f}\{h: h \cap O \neq \varnothing\}$ is the occurrence proposition for $O$, true in $h$ iff $h \in H_{\langle O\rangle} . H_{\langle O\rangle}$ is provably consistent.

$e<O$ is to be read universally: $\forall e_{1}\left[e_{1} \in O \rightarrow e<e_{1}\right]$.

$\mathbf{I}<O$ is also universal: $\forall e[e \in \mathbf{I} \rightarrow e<O]$.

Fact: Since histories are closed downward, if $e<O$ then $H_{\langle O\rangle} \subseteq H_{(e)}$, and if $\mathbf{I}<O$ then $H_{\langle O\rangle} \subseteq H_{[\mathbf{I}]}$.

Outcome chains represent a piece of a "world line." But why must an outcome chain be lower-bounded? The idea is both trivial and critical: Because if a chain has no lower bound, if, that is, it stretches "all the way down" in such a way that nothing comes before it, then there is nothing of which it could be an outcome. It could therefore not figure in any transition event.

The way that BST theory thinks of an outcome chain $O$ is special: The core idea is its coming to be, its getting started or beginning. This comes out in the occurrence proposition that we assign to $O$, namely, the set of histories $H_{\langle O\rangle}$ overlapping $O$, which is exactly the set of histories in which $O$ begins to be (since histories are closed downward).

BST postulates that $O$ has always a unique infimum $\inf (O)$, and it is provable that given $e \in h$, there is an $O$ such that $h \cap O \neq \varnothing$ and $e<O$ and $\inf (O)=e$. It is important in BST theory that we keep firmly in mind outcome chains $O$ whose infimum $\inf (O)$ is a proper lower bound of $O$. Such an outcome chain begins to be without that beginning being located at some first point event of the chain. It is the way that, in the case of ball beginning to move, there is a transition from rest to motion: The motion begins without there being a first instant of motion. 
Scattered outcome events. Outcome chains are theoretically important, but too special. For the second position of a transition event, $\mathbf{I} \longrightarrow$, we need to consider outcome events that are scattered in either a time-like or a space-like way.

D6. $\mathbf{O}$ is a scattered outcome event $\leftrightarrow_{d f} \mathbf{O}$ is a set of outcome chains all of which overlap some one history. I often write $s$-o event for "scattered outcome event." 6

$H_{\langle\mathbf{O}\rangle}=_{d f} \bigcap o \in \mathbf{O} H_{\langle O\rangle}$ is the occurrence proposition for $\mathbf{O}$, true in $h$ iff $h$ $\in H_{\langle\mathbf{O}\rangle}$.

$e<\exists \mathbf{O}$ is to be read existentially: $\exists O[O \in \mathbf{O} \& e<O]$; where, as indicated in D5, " $e<O$ " is to be taken universally.

$\mathbf{I}<{ }_{\exists} \mathbf{O}$ is universal on the left: $\forall e\left[e \in \mathbf{I} \rightarrow e<{ }_{\exists} \mathbf{O}\right]$.

Fact: Since histories are closed downward, if $e<{ }_{\exists} \mathbf{O}$ then $H_{\langle\mathbf{O}\rangle} \subseteq H_{(e)}$, and if $\mathbf{I}<{ }_{\exists} \mathbf{O}$ then $H_{\langle\mathbf{O}\rangle} \subseteq H_{[\mathbf{I}]}$.

So a scattered outcome event is essentially "conjunctive," occurring just in case all of its parts occur.

Scattered outcome events represent outcome events that are not chains. They may be scattered in either a space-like way or a time-like way. A (concrete) birthday party is like that. By saying that such a scattered outcome event occurs, we mean that every scattered part of it occurs. But what are its "parts"? Having seen the need for outcome chains whose infima are proper, we shall lose ground if we take the parts of a scattered outcome event to be point events. It is better to take its parts to be outcome chains, which gives us a more refined meaning for the part beginning to be. This accounts for our definition of a scattered outcome event $\mathbf{O}$ as a set of outcome chains subject to one condition: There must be a history in which each part (i.e., each member) begins to be. Then the occurrence proposition $H_{\langle\mathbf{O}\rangle}$ for $\mathbf{O}$, namely $\bigcap_{o \in \mathbf{O}} H_{\langle O\rangle}$, is bound to be consistent. In this way we can both represent arbitrarily complicated scattered outcome events while still tying their occurrence to beginning-to-be.

It is, however, notable that we do not require that an s-o event $\mathbf{O}$ have a common lower bound for its parts. Each part of $\mathbf{O}$ individually must have a lower bound so that each part can be an outcome of something, but that requirement is scattered among the individual parts. Still, in most intuitively manageable examples such as the birthday party, there will be a common lower bound. The only counterexamples would have to be "infinitely" scattered in either a time-like or a space-like way, and would probably be of only technical interest.

\footnotetext{
${ }^{6}$ Scattered outcome events were called just "outcome events" in Belnap 2002a; the adjective "scattered" was introduce in Belnap 2002b.
} 
S-o events are sets of sets. Since a unit set $\{e\}$ of a point event $e$ is itself an outcome chain, it is a possibility in accord with definitions that a certain s-o event could be a set all of whose members are such unit sets. In this uninteresting case, the occurrence proposition $H_{\langle\mathbf{O}\rangle}$ for the scattered outcome event $\mathbf{O}$ is exactly the same as the occurrence proposition $H_{[\mathbf{I}]}$ for the initial event $\mathbf{I}=\{e:\{e\} \in \mathbf{O}\}$. The extra set-theoretical layer pays off only in more interesting cases.

Disjunctive outcome events. The third type of outcome event needed for a theory of causation is the disjunctive outcome event. In defining the type, we go up yet another set-theoretical level.

D7. $\breve{O}$ is a disjunctive outcome event $\leftrightarrow_{d f} \breve{\mathbf{O}}$ is a set of pairwise inconsistent s-o events (a set of sets of sets). I sometimes write that $\breve{\mathbf{O}}$ is a $d$-o event.

$H_{\langle\breve{\mathbf{O}}\rangle}=_{d f} \bigcup_{\mathbf{O} \in \breve{\mathbf{O}}} H_{\langle\mathbf{O}\rangle}$ is the occurrence proposition for $\breve{\mathbf{O}}$, true in $h$ iff $h$ $\in H_{\langle\breve{\mathbf{O}}\rangle}$.

$e<\forall \exists \breve{\mathbf{O}}={ }_{d f} \forall \mathbf{O}\left[\mathbf{O} \in \breve{\mathbf{O}} \rightarrow e<{ }_{\exists} \mathbf{O}\right]$.

$\mathbf{I}<_{\forall \exists} \breve{\mathbf{O}}={ }_{d f} \forall e\left[e \in \mathbf{I} \rightarrow e<_{\forall \exists} \breve{\mathbf{O}}\right]$.

Fact: Since histories are closed downward, if $e<_{\forall \exists} \breve{\mathbf{O}}$ then $H_{\langle\breve{\mathbf{O}}\rangle} \subseteq H_{(e)}$, and if $\mathbf{I}<_{\forall \exists} \breve{\mathbf{O}}$ then $H_{\langle\breve{\mathbf{O}}\rangle} \subseteq H_{[\mathbf{I}]}$.

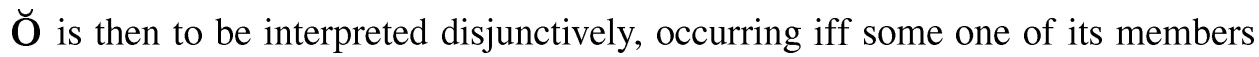
occurs. A d-o event can represent "the birthday party" in a semi-generic sense in which it can occur only in virtue of the occurrence of (exactly) one of its members or "instances." You can "make up" a d-o event any way that you wish and then call it a "single" d-o event. But what you are making up is a set of pairwise disjoint s-o events the occurrence of exactly one of which is to be tantamount to the occurrence of the "single" d-o event. I call d-o events "semi-generic" because the idea is not intended to cover e.g. the generic concept of "birthday party." To compare with ideas of modal logic, we may say that a d-o event is more like an "individual concept" than like an intensional property. D-o events correspond in their spirit to the branching-time events $\mathrm{E}$ of von Kutschera 1993, e of Xu 1997, and $\varepsilon$ of Wölfl 2002. What is the similarity among the branching-time events $E$ of von Kutschera, e of Xu, $\varepsilon$ of Wölfl, and the disjunctive outcome events $\breve{O}$ here? This: Each is defined as a set of more basic event-like entities at most one of which can "occur" in a given history. Each of these publications contains extended analyses and telling examples. For comparisons, generalizations, and deepened analysis of events in branching time, as well as for conceptual suggestions about such events that may have application in branching space-times, see especially Wölfl 2002. 


\subsection{Ontology: transition events}

A transition event is always an ordered pair of an initial event and an outcome. ${ }^{7}$ We shall be considering transition events of the following kinds: $\mathbf{I} \longmapsto O, \mathbf{I} \longmapsto \mathbf{O}$, and $\mathbf{I} \longmapsto \breve{\mathbf{O}}$, and, as special cases, the same three with " $e$ " for "I." The following convention permits a little extra brevity in speaking of these matters.

D8. Let $\bar{I}$ range over two kinds of initial events $e$ and $\mathbf{I}$, and let $\bar{O}$ range over three kinds of outcome events $O, \mathbf{O}$, and $\breve{\mathbf{O}}$.

Let $H_{\bar{I}}$ and $H_{\bar{O}}$ be the respective occurrence propositions of $\bar{I}$ and $\bar{O}$.

In every case of a transition event, $\bar{I} \longmapsto \bar{O}$, we wish there to be a "dropping off" of histories, so that $H_{\bar{O}} \subseteq H_{\bar{I}}$. By the facts stated in connection with definitions D6, D6, and D7, we may guarantee this if we require that the initial $\bar{I}$ of the transition be prior to the outcome $\bar{O}$ of the transition in the "appropriate" sense.

D9. $e[\mathbf{I}]$ is appropriately prior to $O, \mathbf{O}$, or $\breve{\mathbf{O}}$ respectively according as $e<O$ $[\mathbf{I}<O], e<_{\exists} \mathbf{O}\left[\mathbf{I}<_{\exists} \mathbf{O}\right]$ or $e<_{\forall \exists} \mathbf{\mathbf { O }}\left[\mathbf{I}<_{\forall \exists} \mathbf{O}\right]$.

Each of $e \longmapsto O, e \longmapsto \mathbf{O}, e \longmapsto \breve{\mathbf{O}}, \mathbf{I} \longmapsto O, \mathbf{I} \longmapsto \mathbf{O}$, and $\mathbf{I} \longmapsto \breve{\mathbf{O}}$ is a transition event iff its initial is appropriately prior to its outcome.

Fact: If $\bar{I} \longmapsto \bar{O}$ is a transition event, then $H_{\bar{O}} \subseteq H_{\bar{I}}$.

A transition event $\bar{I} \longmapsto \bar{O}$ is contingent iff $H_{\bar{O}} \subset H_{\bar{I}}$.

A paradigm example of a transition event is a choice. Before the choice there is no choice, and after the choice there is no choice. So when is the choice? Bad question: A choice, like any transition event, has no "simple location" (Whitehead). You can locate its initial in the causal order, and you can locate its outcome in the causal order, and having done that, you have done all that you can do. When a choice is made, something happens, but "when" it happens can only be described by giving separate "whens" to its initial and to its outcome. ${ }^{8}$

A transition event, like any event, can occur or not occur. What, then, is the occurrence proposition for a transition event? A good guess would be that it should be an "and then" proposition: First the initial occurs and then the outcome occurs. ${ }^{9}$ It turns out, however, that BST theory makes a different and perhaps surprising choice: The occurrence proposition for a transition event is the "material implication" to the effect that if the initial occurs then the outcome occurs.

\footnotetext{
${ }^{7}$ Transition events are used heavily in Xu's account of causation.

${ }^{8}$ Exactly the same holds if you think of quantum mechanics and replace "choice" by "measurement."

9"And then" is the proper reading for the state transitions pTq of von Wright 1963.
} 
D10. Let $\bar{I} \longmapsto \bar{O}$ be a transition event of one of the types allowed by $\mathrm{D} 9$, and let $H_{\bar{I}}$ and $H_{\bar{O}}$ be the occurrence propositions defined for $\bar{I}$ and $\bar{O}$ respectively. Then $H_{(\bar{I} \mapsto \bar{O})}={ }_{d f}-H_{\bar{I}} \cup H_{\bar{O}}$ is the occurrence proposition for $\bar{I} \longmapsto \bar{O}$, true in $h$ iff $h \in H_{(\bar{I} \hookleftarrow \bar{O})}$, hence iff, if $h \in H_{\bar{I}}$ then $h \in H_{\bar{O}}$.

The final "if-then" must be truth functional. Usually in "ordinary language" applications the negation of a "material implication" "if $A$ then $B$ " seems wrong; this is of course one of the motivations for various treatments of counterfactual conditionals, to say nothing of relevance logic. Here, however, there is a better fit: For the transition $\bar{I} \longmapsto \bar{O}$ not to occur is for the initial to occur and then for some other outcome of $\bar{I}$ to occur instead. It is not merely for the outcome $\bar{O}$ not to occur. For instance, if you understand a particular choice as a transition from a particular occasion of indecision to a settled state of having selected the tuna, then for that transition event not to occur is for the chooser to have chosen otherwise from that very same occasion of indecision. For the non-occurrence of the transition event, it does not suffice that the chooser was never born-although that would certainly be sufficient for the non-occurrence of the tuna-selection outcome. Furthermore, we naturally say that a transition $\bar{I} \longmapsto \bar{O}$ is (historically) "noncontingent" when the initial already deterministically guarantees the outcome, that is, when $H_{\bar{O}}$ is not merely a subset of $H_{\bar{I}}$ (as must always be the case by the settledness of the past), but identical to $H_{\bar{O}}$. In that case one notices that the transition-event occurrence proposition rightly turns out to be the universal proposition $\left(-H_{\bar{I}} \cup H_{\bar{O}}\right)=$ $\left(-H_{\bar{O}} \cup H_{\bar{O}}\right)=H i s t$, which is a paradigm of historical noncontingency.

One should not be deeply interested in transition events whose occurrence in $h$ is merely a matter of the initial not occurring in $h$, and so it is good to mark this by saying that the transition event occurs vacuously in $h$. I don't suppose that "vacuous occurrence" is a helpful notion either for initial events or for outcome events, but it does seem to have a role in the theory of transition events. ${ }^{10}$

\subsection{Propositional language applied to events}

Aside from special cases introduced below as "primary" or "basic," we have enough kinds of events. Here I simply point out that BST theory makes natural sense of applying "propositional talk" to events.

D11. Every one of the properties of and relations between propositions that we defined in $\S 3.2$ can now sensibly be applied to events by way of the occurrence propositions that we have defined.

\footnotetext{
${ }^{10}$ I personally find it strange to rely on the "material-implication" understanding of the conditional, if $h \in H_{\bar{I}}$ then $h \in H_{\bar{O}}$. It seems, however, exactly what the theory requires. The choice certainly has nothing to do with views on the meaning of conditionals in everyday speech.
} 
A couple of examples should suffice. The first makes obvious the relevance to causation of a rigorously defined application of propositional language to events.

1. To say that a certain initial event $\bar{I}$ is a necessary condition of a certain outcome event $\bar{O}$ is to say that there is the appropriate relation between their occurrence propositions: $H_{\bar{O}} \subseteq H_{\bar{I}}$. It is then no big calculation to see that for each transition event $\bar{I} \longmapsto \bar{O}$, the occurrence of the initial is an historically necessary condition of the occurrence of the outcome.

2. A set of events of various specified types (perhaps some are initial events and some are outcome events) is jointly consistent iff the set of their occurrence propositions is jointly consistent in the sense of $\$ 3.2$. This kind of example, not of further note in this essay, is important in understanding what it can mean, for instance, to ask if, in a quantum-mechanical experiment, the joint setting-up of two space-like related measurements (settings-up are initial events) does or does not prohibit a certain joint outcome; see Szabo and Belnap 1996 for an application of this idea.

\section{Causae causantes}

I now turn to causae causantes, which I eventually show to be inus conditions. The proposal is to identify causae causantes neither with initial events nor with outcome events, but instead with certain transition events. A transition event is "where" something happens; it is "where" there is a transition from (to use Mackie's language) unfixity to fixity. (The shudder quotes remind us that for transition events there is no "simple location.") An effect may also be a transition event. If I receive a letter that, by choice or chance, might or might not have been sent, then there is for me somewhere on my world line a transition from "might not receive letter" to "settled that I will receive letter," but that transition event is purely passive, a mere effect. The causa causans in this case is along the world line of the sender, not the receiver.

Let me indicate one dead end: The kinds of transitions that we consider are modal transitions. They are not merely state transitions. If at a certain moment, for example, there is a (real) possibility of motion, then "remaining at rest" would cut off certain possibilities and thus be a transition event of a kind that is the object of our investigation, even though there is no "change of state."

\subsection{Causae causantes are basic primary transition events}

I speak of a single causa causans, but of course a great many causae causantes must be cooperating in order to produce the receive-letter effect. Our first task, 
however, is to characterize a single causa causans as a certain special kind of transition.

All of the earlier BST essays have taken as fundamental the transition from a single point event $e$ to one of its immediate possibilities, to a possibility, that is, that is realized, if at all, in the immediate future of $e$. The outcome of such a transition can be represented in either of two (equivalent) ways, as a proposition defined in terms of undividedness, or as a scattered outcome event consisting of outcome chains all of which begin immediately after $e .{ }^{11}$ Here are the definitions,

D12. $h_{1}$ is undivided from $h_{2}$ at $e$, written $h_{1} \equiv_{e} h_{2}, \leftrightarrow_{d f} \exists e_{1}\left[e<e_{1} \& e_{1} \in\left(h_{1} \cap\right.\right.$ $\left.h_{2}\right)$.

Much-used fact: Undividedness is an equivalence relation on $H_{(e)}$.

For $e \in h, \Pi_{e}\langle h\rangle=_{d f}\left\{h_{1}: h_{1} \equiv_{e} h\right\}$.

$\Pi_{e}={ }_{d f}\left\{\Pi_{e}\langle h\rangle: h \in H_{(e)}\right\}$. Fact: $\Pi_{e}$ is a partition of $H_{(e)}$.

In a mouthful, a member $\Pi_{e}\langle h\rangle$ of $\Pi_{e}$ is said to be a basic primary propositional outcome of $e$. (I also retain the language of atomic immediate possibility at e.)

$\Pi_{e}\langle h\rangle$ is the set of histories that are undivided from $h$ at $e$, and thus, since histories that are undivided at $e$ do not divide until properly after $e$, histories in $\Pi_{e}\langle h\rangle$ must belong together in the same atomic immediate possibility at $e$. Unless $\Pi_{e}\langle h\rangle$ is trivially identical to $H_{(e)}, \Pi_{e}\langle h\rangle$ is a proposition that is neither settled true (see Definition 7-1(4)) nor settled false at $e$, but is settled one way or the other at every point event $e_{1}$ such that $e<e_{1}$.

According to the other representation, a transition fit to be a causa causans is from an initial point event to a scattered outcome event, rather than to a proposition. What makes the s-o event $\mathbf{O}$ an immediate outcome of $e$ is that for every outcome chain $O \in \mathbf{O}, \inf (O)=e$. There are many such chains; we cut them down to a particular scattered outcome event by taking into account their relation to histories, as follows.

D13. For $e \in h, \Omega_{e}\langle h\rangle=d f\{O: \inf (O)=e \& h \cap O \neq \varnothing\}$.

$\Omega_{e}={ }_{d f}\{\mathbf{O}: \mathbf{O}$ is a basic primary scattered outcome of $e\}$.

Each member $\Omega_{e}\langle h\rangle$ of $\Omega_{e}$ is a basic primary scattered outcome of $e$.

The members of $\Omega_{e}\langle h\rangle$ evidently begin in the immediate future of $e$, so that between $e$ and members of $\Omega_{e}\langle h\rangle$ there is no "room" for "influences" from the past.

\footnotetext{
${ }^{11}$ Only the first of these representations occurs in earlier BST essays.
} 
There is some elaboration of this thought in earlier BST essays. The "scattering" takes some of these outcome chains into remote corners of Our World. ${ }^{12}$

Since $\Omega_{e}\langle h\rangle$ is a scattered outcome event, $H_{\left\langle\Omega_{e}\langle h\rangle\right\rangle}$ makes sense as a proposition.

4-1 FACT. (Interchangeability of $\Omega_{e}\langle h\rangle$ and $\Pi_{e}\langle h\rangle$ )

- An easy fact is that the occurrence proposition $H_{\left\langle\Omega_{e}\langle h\rangle\right\rangle}$ for $\Omega_{e}\langle h\rangle$ is the same proposition as $\Pi_{e}\langle h\rangle$.

- Of deeper significance is that even though occurrence propositions do not in general determine events, in this special case, when we are given not only the proposition, but also $e$, we have that if $H_{\left\langle\Omega_{e}\left\langle h_{1}\right\rangle\right\rangle}=H_{\left\langle\Omega_{e}\left\langle h_{2}\right\rangle\right\rangle}$ then $\Omega_{e}\left\langle h_{1}\right\rangle=$ $\Omega_{e}\left\langle h_{2}\right\rangle$. That is, $\left.H_{\left\langle \_\right.}\right\rangle$is a natural one-one correspondence between the basic primary scattered outcomes $\Omega_{e}$ of $e$ and the basic primary propositional outcomes $\Pi_{e}$ of $e .^{13}$

I use these two equivalent representations of atomic immediate outcomes almost interchangeably even though $\Pi_{e}\langle h\rangle$ is a proposition and $\Omega_{e}\langle h\rangle$ is a scattered outcome event. The chief place in which I rely on the interchange is in the idea of a causa causans:

D14. $e \longmapsto \Omega_{e}\langle h\rangle$ is a basic primary transition event.

$e \longmapsto \Pi_{e}\langle h\rangle$ is a basic primary propositional transition.

Both $e \hookrightarrow \Omega_{e}\langle h\rangle$ and $e \longmapsto \Pi_{e}\langle h\rangle$ may be called basic primary transitions. Indeed, sometimes I even call $e \longmapsto \Pi_{e}\langle h\rangle$ a transition "event" even though its outcome is propositional.

A causa causans or originating cause is by definition any basic primary transition, except that we exclude the trivial transition events, those such that $\Pi_{e}\langle h\rangle=H_{(e)}$.

I should think that $e \longmapsto \Omega_{e}\langle h\rangle$ comes first conceptually just because it is an "event" in the proper sense. In passages below, however, I most often write $e \longmapsto \Pi_{e}\langle h\rangle$ for two reasons: (1) habit, and (2) its use avoids the visually difficult combination " $e$ $\longmapsto H_{\left\langle\Omega_{e}\langle h\rangle\right\rangle}$."

\footnotetext{
${ }^{12}$ Let us note explicitly that $\bigcup \Omega_{e}\langle h\rangle$ need not be internally "historically connected" as required by Definition 2.1(iii) of Xu 1997. You can see this if you think of a pair $O_{1}$ and $O_{2}$ of members of $\Omega_{e}\langle h\rangle$ each of which is the path of a light ray in an imagined two-dimensional Minkowski diagram, and each going in an opposite direction from the other. If you pick two point events $e_{1} \in O_{1}$ and $e_{2}$ $\in O_{2}$, you will find that $e_{1}$ and $e_{2}$ have no common lower bound in $\bigcup \Omega_{e}\langle h\rangle$.

${ }^{13}$ It by no means follows that, if $H_{\left\langle\Omega_{e_{1}}\left\langle h_{1}\right\rangle\right\rangle}=H_{\left\langle\Omega_{e_{2}}\left\langle h_{2}\right\rangle\right\rangle}$ then $\Omega_{e_{1}}\left\langle h_{1}\right\rangle=\Omega_{e_{2}}\left\langle h_{2}\right\rangle$. You must hold $e$ constant.
} 
The choices by agents that are discussed in Belnap et al. 2001 can be identified as immediate transition events, without any interval between initial and outcome. Though spelled out in terms of branching time rather than in terms of branching space-times, agentive choices are causae causantes.

\subsection{Causae causantes of an outcome chain}

Given the proposed notion of a causa causans or originating cause as a certain kind of entity (namely, a basic primary transition), and given that we are after inus conditions of effects or results, we need to fix the relational idea of an originating cause of something. We shall need to look at causae causantes of outcome events of various kinds, and of transition events. I start with the simplest and also most fundamental in BST theory: Let us think of an effect or result represented by an outcome chain $O$. (Later I enter the modifications needed for $\mathbf{O}$ and $\breve{\mathbf{O}}$, and for transition events.) Which causae causantes are causae causantes of $O$ ? Position yourself at some point event $e_{1}$ such that some part of $O$ is in its causal past, so that it is a settled fact at $e_{1}$ that $O$ occurs $\left(H_{\left(e_{1}\right)} \subseteq H_{\langle O\rangle}\right)$. Represent some most-detailed "otherwise" by a history $h$, a history in which $O$ does not occur. Then the prior choice postulate of BST theory assures us that there is a point event $e$ in the past of $O$ at which there is a split between the continued possibility of $O$ and the continued possibility of $h$. That is, $e$ is a "cause-like locus" for $O$. Before continuing, let us pause to enter the needed definitions, which are discussed and motivated in earlier BST essays.

D15. $h_{1}$ is separated from $h_{2}$ at $e$, written $h_{1} \perp_{e} h_{2}, \leftrightarrow_{d f} e$ is maximal in $h_{1} \cap h_{2}$. $h_{1}$ is separated from $H$ at e, written $h_{1} \perp_{e} H, \leftrightarrow_{d f} \forall h_{2}\left[h_{2} \in H \rightarrow h_{1} \perp_{e} h_{2}\right]$. $H_{1}$ is separated from $H_{2}$ at $e$, written $H_{1} \perp_{e} H_{2}, \leftrightarrow_{d f}$ every history in $H_{1}$ is separated at $e$ from every history in $H_{2}$.

D16. $e$ is a basic cause-like locus for $O$ iff $h \perp_{e} H_{\langle O\rangle}$ for some $h$.

The plan is to identify each initial of a causa causans with a basic cause-like locus of $O .{ }^{14}$ Causae causantes of $O$ are precisely those that make a difference to whether or not $O$ occurs. ${ }^{15}$ The trouble is that if there is funny business in the sense

\footnotetext{
${ }^{14}$ Given my repeated insistence that causae causantes have no "simple location," it should be obvious that "locus" in the phrase "cause-like locus" references only the locus of an initial of something that is cause-like.

${ }^{15}$ Of course there are a lot of them. Some are salient, some are not, to some we have epistemological access, to some not, some are more recent, some lie in the distant past, some are important, some are trivial. About none of that, however, can BST theory be informative. I repeat: BST, although resolutely objective, is not a theory of everything.
} 
of $\S 4.3$, then some of these may not lie in the past of $O$. I approach the difficulty gingerly by defining the set of cause-like loci that seem easiest to understand: the set of past cause-like loci of $O$. At least the prior choice postulate of BST theory promises us one of these for every $h \notin H_{\langle O\rangle}$.

D17. $p c l(O)$, read as either the set of past cause-like loci for $O$, or, more bravely, as the set of past causal loci for $O,=_{d f}\left\{e: e<O \& \exists h\left[h \perp_{e} H_{\langle O\rangle}\right]\right\}$.

The question is, which of the many basic primary outcomes of $e$ should be considered in defining the idea of a causa causans of $O$ ? That may turn out to be a hard question when a basic cause-like locus $e$ for $O$ (D16) is not in the past of $O$, a situation that can definitely arise in Our World to the extent that there is EPR-like funny business as described in $\S 4.3$. In the present circumstances, however, we are thinking only about members of $\operatorname{pcl}(O)$, which by brute definition lie in the past of $O: e<O$. It is easy to calculate that $e<O$ implies that the occurrence of $O$ is consistent with exactly one basic primary outcome of $e$. Why easy? Because $O$ is a chain and because histories are closed downward, any two histories in the occurrence proposition $H_{\langle O\rangle}$ are bound to share a point event $e_{1}$ such that $e<e_{1}$, so that by definition the two histories are undivided at $e$, so that they must belong to the same member of $\Pi_{e}$.

So at this point we have an initial $e \in \operatorname{pcl}(O)$, and we want a basic primary transition $e \longmapsto \ldots$ as a causa causans of $O$. All we need is one more piece of notation, a function of $e$ and $O$ when $e<O$, in order clearly to define "causa causans of $O . "$

D18. When $e<O, \Pi_{e}\langle O\rangle$, read the projection of $O$ onto $e,{ }_{d f}$ the unique member of $\Pi_{e}$ that is consistent with $H_{\langle O\rangle}$. Alternatively and equivalently, $\Pi_{e}\langle O\rangle$ ${ }_{d f}\left\{h: h \equiv_{e} H_{\langle O\rangle}\right\}$.

Fact: On either of these definitions, if $e<O$ then $\left(\Pi_{e}\langle O\rangle \in \Pi_{e}\right.$ and $H_{\langle O\rangle} \subseteq$ $\left.\Pi_{e}\langle O\rangle\right)$.

It is easy to see that the occurrence proposition $H_{\langle O\rangle}$ for $O$ says that $O$ "occurs" in $h$ in the sense of beginning to be in $h$, but it is not so easy to understand $\Pi_{e}\langle O\rangle$ in an intuitive way. One must keep in mind that $\Pi_{e}\langle O\rangle$, since it belongs to $\Pi_{e}$, is an immediate possibility at $e$. $O$, however, is a more distant possibility, and, as everyone knows, there can be many a slip between $e$ and a distant $O$. So what, in terms of $O$, happens immediately at $e$ when our world takes a turn toward $\Pi_{e}\langle O\rangle$ ? Nothing more nor less than this: The occurrence of $O$ is kept possible. In contrast every other member of $\Pi_{e}$ renders $O$ henceforth impossible. The transition is not to the occurrence of $O$ (which is expressed by $H_{\langle O\rangle}$ ), but to the continued possibility of its occurrence (which is expressed by $\Pi_{e}\langle O\rangle$ ). This formulation puts weight on 
"kept possible" since (1) $O$ is possible at $e$ itself and (2) the truth in $h$ of $\Pi_{e}\langle O\rangle$ by no means implies that $O$ stays possible for long. All we are given is that $O$ remains possible in the perhaps very short immediate future of $e$. So in words that are carefully chosen although hard to process, $\Pi_{e}\langle O\rangle$ (when $e<O$ ) comes to this: At $e$ it is possible that $O$ occurs, but furthermore, at $e$ it so happens that it will be possible that $O$ occurs. In Definition 7-1(13) the meaning of these words is spelled out semantically as a complex object-language construction.

Now it is entirely in order to define the causae causantes of $O$ as the appropriate set of basic primary transition events whose initials belong to $\operatorname{pcl}(O)$ :

D19. A causa causans of $O$ is defined as any basic primary transition $e \longmapsto \Pi_{e}\langle O\rangle$ such that $e \in \operatorname{pcl}(O)$. In symbols:

$c c(O)$, read the set of causae causantes of $O,=d f\left\{e \longmapsto \Pi_{e}\langle O\rangle: e \in p c l(O)\right\}$. I use "originating cause" as a synonym of "causa causans."

Observe that the transition $e \longmapsto \Pi_{e}\langle O\rangle$ is bound to be contingent when $e \in \operatorname{pcl}(O)$. It is furthermore well to note explicitly that each causa causans of $O$ lies "in the past" of $O$ in a plain and definite sense: The initial of the causa causans is in the causal past of $O$. Since transition events, to repeat a mantra, have no "simple location," some such subtlety is to be expected.

\subsection{No funny business}

The goal is to show that causae causantes are inus conditions in the sense of Quasidefinition 1-1. There is, however, a complication with which we must deal. BST permits "funny business," that is, correlations between space-like separated primary propositional transitions (to give one of four equivalent definitions). Such funny business seems to be a consequence of quantum mechanics (Bell, EPR). Causal talk is at the very least much more difficult in the presence of funny business. It is not enough to build pastness into the idea of causae causantes by inserting pastness as part of the definition $\mathrm{D} 17$ of $p c l(O)$. I am going to enter an assumption for the scope of this essay that there is no funny business. I do not know to what extent the ideas of this essay survive in the presence of funny business.

True, this demands that for present purposes we take pastness to be an essential part of the fabric of causation. What justifies this? The belief that in the presence of funny business, we cannot trust our ideas of causality as much as we should like; and in the absence of funny business, the adjective "past" is redundant: If $e$ is cause-like with respect to $O$, then "no funny business" guarantees that $e$ is in the past of $O$, since it says that all cause-like loci for $O$ lie in its past. In a study 
of causality (or cause-likeness) that considered funny business, we should have to be more circumspect. For the present, however, keeping straight about causation even without funny business seems a sufficient challenge, so that I hope I shall be forgiven for "looking where the light is bright."

Stating the assumption requires a little additional terminology the significance of which is explained in earlier BST essays.

D20. Point events are space-like-related iff they are distinct, not causally ordered, and share a history. $\mathbf{I}_{1}$ SLR $\mathbf{I}_{2}$ means that every point event in $\mathbf{I}_{1}$ is space-like related to every point event in $\mathbf{I}_{2}$.

D21. Provided $\mathbf{I} \subseteq h, \Pi_{\mathbf{I}}\langle h\rangle={ }_{d f} \bigcap_{e \in \mathbf{I}} \Pi_{e}\langle h\rangle$.

$h_{1}$ is separated from $H$ at $\mathbf{I}$, written $h_{1} \perp_{\mathbf{I}} H, \leftrightarrow_{d f} \forall h_{2}\left[h_{2} \in H \rightarrow \exists e[e \in \mathbf{I}\right.$ and $\left.h_{1} \perp_{e} h_{2}\right]$.

$h$ is relevantly separated from $H$ at $\mathbf{I}$, written $h \perp_{\mathbf{I}} H, \leftrightarrow_{d f} h \perp_{\mathbf{I}} H$ and $\forall e[e$ $\in \mathbf{I} \rightarrow \exists h_{1}\left[h_{1} \in H\right.$ and $\left.\left.h \perp_{e} h_{1}\right]\right]$.

$\mathbf{I}$ is a cause-like locus for $\mathbf{O} \leftrightarrow d f \exists h\left[h \perp_{\mathbf{I}} H_{\langle\mathbf{O}\rangle}\right]$.

This means, roughly, that before and at the initial $\mathbf{I}$ each of $h$ and the occurrence of $\mathbf{O}$ are possible, but after $\mathbf{I}$ at least one of these options is no longer possible. Thus, $\mathbf{I}$ is a "decision point" at which it is decided whether or not the occurrence of $\mathbf{O}$ remains a possibility for the future. In the "basic" case of D16, the initial is a single point event $e$. Here is the assumption stated in four ways that are explained and proved equivalent in earlier BST essays.

4-2 Assumption. (No funny business) Our World may be indeterministic, but for the course of this essay we assume that it is free of EPR-like funny business in any and all of the following interchangeable senses:

1. No some-cause-like-locus-not-in-past funny business (every cause-like locus for an outcome event lies in its past). If an initial event $\mathbf{I}$ is a cause-like locus for an s-o event $\mathbf{O}$, then $\mathbf{I}$ lies in the past of $\mathbf{O}$ in a weak sense: For some $e$ $\in \mathbf{I}, e<{ }_{\exists} \mathbf{O}$.

2. No primary modal-correlation funny business (immediate outcomes of spacelike related initials are always modally independent). If $\mathbf{I}_{1}$ SLR $\mathbf{I}_{2}$ and $\mathbf{I}_{1} \subseteq$ $h_{1}$ and $\mathbf{I}_{2} \subseteq h_{2}$, then $\Pi_{\mathbf{I}_{1}}\left\langle h_{1}\right\rangle \cap \Pi_{\mathbf{I}_{2}}\left\langle h_{2}\right\rangle \neq \varnothing$.

3. Never absence-of-prior-screener-off funny business (always a prior screeneroff). If $\left(H_{\left\langle\mathbf{O}_{1}\right\rangle} \cap H_{\left\langle\mathbf{O}_{2}\right\rangle}\right)=\varnothing$ then $\exists e\left[e<{ }_{\exists} \mathbf{O}_{1}\right.$ and $e<{ }_{\exists} \mathbf{O}_{2}$ and $\forall h[e \in h \rightarrow$ $\left(\Pi_{e}\langle h\rangle \cap H_{\left\langle\mathbf{O}_{1}\right\rangle}=\varnothing\right.$ or $\left.\left.\left.\left.\Pi_{e}\langle h\rangle \cap H_{\left\langle\mathbf{O}_{2}\right\rangle}=\varnothing\right)\right]\right]\right]$. 
4. Never absence-of-common-prior-cause-like-locus funny business (always a common prior cause-like locus). If $\left(H_{\left\langle\mathbf{O}_{1}\right\rangle} \cap H_{\left\langle\mathbf{O}_{2}\right\rangle}\right)=\varnothing$ then $\exists e\left[e{ }_{\exists} \mathbf{O}_{1}\right.$ and $e<{ }_{\exists} \mathbf{O}_{2}$ and $\left.\left.H_{\left\langle\mathbf{O}_{1}\right\rangle} \perp_{e} H_{\left\langle\mathbf{O}_{2}\right\rangle}\right]\right]$.

The precise significance of funny business vs. no funny business for causation is by no means clear. Maybe one should promote "cause-like locus" to "causal locus," and maybe not. It is an assumption of the present investigation that our run-of-the-mill thoughts about causation always involve pastness, so that we may well not know exactly what to say about a cause-like locus for $O$ that is not in the past of $O$. That is our justification, such as it is, for Assumption 4-2. Its most immediate consequence is this:

The clause " $e<O$ " in the definition $\mathrm{D} 17$ of $p c l(O)$ is redundant.

That is, however, hardly the chief sticking point, since we could and did avoid that use of Assumption 4-2 by simply defining pastness into the concept of $\operatorname{pcl}(O)$. Look for our use of the assumption in what follows.

\section{Causae causantes and inns and inus conditions}

We have isolated the idea of causae causantes. In this section we show that causae causantes are inus conditions. We will be considering the originating causes of six kinds of "effects": outcome chains $O$, scattered outcome events $\mathbf{O}$, disjunctive outcome events $\breve{\mathbf{O}}$, and transition events $\mathbf{I} \longmapsto O, \mathbf{I} \longmapsto \mathbf{O}$, and $\mathbf{I} \longmapsto \breve{\mathbf{O}}$. So far we have only defined "originating cause of" for outcome chains $O$ (D19), and we shall bring to completion the analysis of this the simplest case before considering the other five.

An outcome chain $O$ may be considered "atomic" in the sense that it can have only one causal situation, which is intended to mean that its settled causal past is independent of the history of its occurrence. Full inus conditions in the sense of Quasi-definition 1-1, however, are fit only for disjunctive events that, in a sense to be made clear (D22) can be causally situated in more than one way. It is the disjunctive element that is caught by "unnecessary" in the outer part of the Mackie phrase of Quasi-definition 1-1, "unnecessary but sufficient condition": Each disjunct of a disjunction is on the face of it an "unnecessary but sufficient condition" for the disjunction. When we get down to outcome chains, however, disjunctiveness disappears, so that in this case we are not really looking for inus conditions. In this case, we are looking for what we will call "inns" conditions:

5-1 QUASI-DEFINITION. (Inns condition) An inns condition of an event is an insufficient but non-redundant part of a necessary and sufficient condition. 

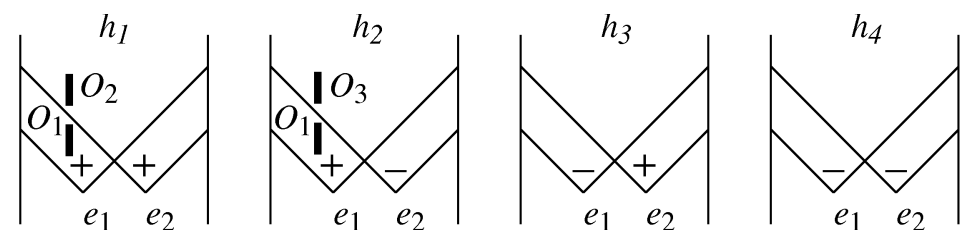

Figure 1: Aristotle's market-goers

In other words, the picture is that $A B C$ is a necessary and sufficient condition for $P$, with the extra claim that the insufficient conjunctive part $A$ is non-redundant. Compare the account Quasi-definition 1-1 of inus conditions.

An inns condition is what is appropriate when a result can be causally situated in only one way, and so we shall be trying to show that causae causantes of $O$ are inns conditions of the occurrence of $O$.

It is critical to the present analysis that we are asking for causes of events rather that of propositions; let us pause to illustrate this point. Consider Figure $1 .{ }^{16}$ Point events $e_{1}$ and $e_{2}$ represent choices by two market-goers in distant villages, "+" representing "go to market" and "-" representing "not go." $O_{1}$ is a portion of the world line of market-goer \#1 at a point before it has become settled for him whether or not market-goer \#2 has chosen to go, whereas $\mathrm{O}_{2}$ is a portion of the world line of \#1 after it has become settled for him that \#2 is going to market. Perhaps at point events in $\mathrm{O}_{2}$ the first market-goer actually encounters the second market-goer. Similarly, $\mathrm{O}_{3}$ is a portion of the world line of \#1 after it has become settled that \#2 does not go to market; perhaps at point events in $O_{3} \# 1$ can actually see that \#2 is not at the market.

The three occurrence propositions for these outcome chains are evidently as follows: $H_{\left\langle O_{1}\right\rangle}=\left\{h_{1}, h_{2}\right\} ; H_{\left\langle O_{2}\right\rangle}=\left\{h_{1}\right\}$; and $H_{\left\langle O_{3}\right\rangle}=\left\{h_{2}\right\}$. Therefore $H_{\left\langle O_{1}\right\rangle}=$ $\left(H_{\left\langle O_{2}\right\rangle} \cup H_{\left\langle O_{3}\right\rangle}\right)$, which makes $H_{\left\langle O_{1}\right\rangle}$ a "disjunctive proposition." Right. But we are not asking for causes of propositions, not even of occurrence propositions. We are asking for causes of events. As such, there is nothing "disjunctive" about $O_{1}-$ it can occur in more than one way (with or without the presence of market-goer \#2), but it can only be causally situated in a single way: Regardless of history of occurrence, the causal situation of the chain $O_{1}$ as encoded by its settled causal past is quite the same. In contrast, if we are interested in the disjunctive event $\breve{\mathbf{O}}=$

\footnotetext{
${ }^{16}$ For help in decoding Figure 1, see the earlier BST essays, and especially note 23 of Belnap $2002 \mathrm{a}$.
} 
$\left\{\left\{O_{2}\right\},\left\{O_{3}\right\}\right\},{ }^{17}$ then we want a story about the $t w o$ ways that it can be causally situated: That disjunctive event has one causal past in $h_{1}$, and an entirely different causal past in $h_{2}$.

This may become a little clearer if I introduce an account of the "causal past" of the three sorts of outcome events.

D22. $J^{-}(O)=_{d f}\{e: e<O\} . J^{-}(\mathbf{O})={ }_{d f} \bigcup O \in \mathbf{O}^{J^{-}}(O)$.

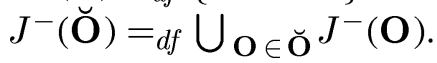

Fact: For $h_{1}, h_{2} \in H_{\bar{O}}, J^{-}(\bar{O}) \cap h_{1}=J^{-}(\bar{O}) \cap h_{2}$ is guaranteed for $\bar{O}$ either an outcome chain $O$ or a s-o event $\mathbf{O}$, but not for a d-o event $\breve{\mathbf{O}}$.

For disjunctive events, the causal situation depends in general on the history of occurrence, but s-o events and outcome chains can be situated in just one way, even though they can occur in more than one way. You cannot make this distinction with propositions alone; you need the events.

\subsection{Inns conditions of outcome chains $O$ : not quite}

In figuring out the inns conditions of $O$, it will be instructive first to try something that is true but is not quite on target.

5-2 FACT. $($ About $p c l(O)) \quad H_{\langle O\rangle}=\bigcap_{e \in p c l(O)} \Pi_{e}\langle O\rangle$.

Fact 5-2 tells us that $O$ occurs in exactly those histories in which all the propositional outcomes $\Pi_{e}\langle O\rangle$ are true. So each member of $\left\{\Pi_{e}\langle O\rangle: e \in p c l(O)\right\}$ is an insufficient part of a necessary and sufficient condition for the occurrence of $O$.

This is in the vicinity of the notion of an "inns" condition (Quasi-definition 5-1). There is, however, a big difference: The " $n$ " in "inus" and the first " $n$ " in inns signifies "non-redundant" rather than "necessary" in the sense of "necessary condition." Of course "necessary" can also mean "non-redundant," but it is not the same idea. For example, suppose one has a conjunction $H_{1} \cap H_{2} \cap H_{3}$ that is necessary and sufficient for the occurrence of $O$. Then each conjunct, say $H_{1}$, is certainly a necessary condition for $O$. It could still be that in the conjunction, the conjunct $H_{1}$ is redundant; for example, it might be that $H_{1}$ is implied by the remaining conjuncts (or some of them) taken together, so that $H_{1}$ could be dropped without loss. Then $H_{1}$ would be a necessary condition of $O$, but not non-redundant. What we would need to show for the inns idea is not just that each $\Pi_{e}\langle O\rangle$ is a necessary condition of $H_{\langle O\rangle}$, but that for each $e_{0} \in \operatorname{pcl}(O), \Pi_{e_{0}}\langle O\rangle$ is non-redundant in

\footnotetext{
${ }^{17}$ The extra set-theoretical level here is included for technical exactness, but is evidently just baggage.
} 
the sense that you cannot leave it out: $\bigcap_{e \in\left(p c l(O)-\left\{e_{0}\right\}\right)} \Pi_{e}\langle O\rangle$ is not sufficient for $H_{\langle O\rangle}$. That would show that the outcome $\Pi_{e_{0}}\langle O\rangle$ of the transition $e_{0} \longmapsto \Pi_{e_{0}}\langle O\rangle$ is non-redundant in the context of all the outcomes. Let's see.

Well, Fact 5-2 tells us nothing about non-redundancy, and indeed the claim to the non-redundancy of $\Pi_{e}\langle O\rangle$ (for $e \in p c l(O)$ ) is false. In fact if $e_{1}<e_{2}$ (both point events belonging to $\operatorname{pcl}(O)$ ), then it is certain that $\Pi_{e_{2}}\langle O\rangle$ implies $\Pi_{e_{1}}\langle O\rangle$, so that the latter is redundant. This is so just by using the "Fact" that is part of D18, and noting that $e_{1}<e_{2}$ implies that if $h_{1} \equiv_{e_{2}} h_{2}$ then $h_{1} \equiv_{e_{1}} h_{2}$. Therefore, if we think of just the set $\left\{\Pi_{e}\langle O\rangle: e \in \operatorname{pcl}(O)\right\}$, we shall find that its members, while being each an insufficient but necessary condition of $O$ as well as part of a jointly sufficient condition for $O$, are not inns conditions since not non-redundant.

\subsection{Inns conditions of outcome chains $O$}

BST permits, however, a better candidate for inns conditions. We have to take into consideration that we are speaking of transition events $e \longmapsto \Pi_{e}\langle O\rangle$ rather than simply their outcomes $\Pi_{e}\langle O\rangle$. Here is the very essence of my suggestion: It is the transition events that are inns conditions of the outcome. ${ }^{18}$

Then the occurrence of each member of $c c(O)$ is not only a necessary condition of the occurrence of $O$, and not only are the occurrences of members of $c c(O)$ jointly sufficient for the occurrence of $O$, but the occurrence of each is nonredundant. Therefore we have an inns condition in the best possible sense.

\section{5-3 THEOREM. (The causae causantes of $O$ are inns conditions)}

1. (Historical) necessity of each causa causans of $O$.

$$
H_{\langle O\rangle} \subseteq \bigcap_{e \in \operatorname{pcl}(O)} H_{\left(e \succ \Pi_{e}\langle O\rangle\right)} .
$$

This says that each causa causans of $O$ is a (historically) necessary condition of $O$.

2. Joint (historical) sufficiency of the causae causantes of $O$.

$$
\bigcap_{e \in \operatorname{pcl}(O)} H_{\left(e \hookrightarrow \Pi_{e}\langle O\rangle\right) \subseteq H_{\langle O\rangle}} .
$$

This says that the causae causantes of $O$ taken jointly suffice for the occurrence of $O$.

3. Non-redundancy of each causa causans of $O$. Suppose that $e_{0} \in \operatorname{pcl}(O)$. Then

\footnotetext{
${ }^{18}$ Mea culpa: The account of the "transition stit" of Belnap et al. 2001 is seriously muddled, using outcomes where it should have used transitions.
} 


$$
\bigcap_{e \in\left(p c l(O)-\left\{e_{0}\right\}\right)} H_{\left(e \hookleftarrow \Pi_{e}\langle O\rangle\right)} \nsubseteq H_{\langle O\rangle} .
$$

This says that each causa causans of $O$ is non-redundant: If any causa causans is omitted, the rest do not suffice for the occurrence of $O$. Perhaps this: Take any causa causans of $O$. If all of the other causae causantes of $O$ were to occur, but the one were not occur, then $O$ would not occur. (That was a subjunctive conditional; see $\S 6$ for a brief discussion.)

Altogether, the theorem says that each causa causans of $O$ is an inns condition of $O$ : Each causa causans of $O$ is an insufficient but non-redundant part of a necessary and sufficient condition for $O$. Technically the following proof is selfcontained, but its style really presupposes some familiarity with earlier BST essays.

Proof. $A d$ (1). In fact, it is easy to show that $H_{\langle O\rangle} \subseteq \Pi_{e}\langle O\rangle$ follows from $e<O$ alone: $H_{\langle O\rangle} \subseteq \bigcap_{e<O} \Pi_{e}\langle O\rangle$. See the "Fact" listed as part of D18.

Ad (2). Show the contrapositive by invoking the prior choice postulate: Choose $h \notin H_{\langle O\rangle}$, and then choose $e$ such that $(a) e<O$ and $(b) h \perp_{e} H_{\langle O\rangle}$. Evidently $e \in \operatorname{pcl}(O)$, so that it suffices to show that $h \notin H_{\left(e \hookrightarrow \Pi_{e}\langle O\rangle\right)}$, for which we need that $(y) h \in H_{(e)}$ and $(z) h \notin \Pi_{e}\langle O\rangle$. But (y) follows immediately from (b). Further, since (a), we know by D18 that $\Pi_{e}\langle O\rangle \subseteq H_{\langle O\rangle}$, so that $h \perp_{e} \Pi_{e}\langle O\rangle$ follows from $(b)$, so $(z)$, which finishes this part of the proof.

Ad (3). Suppose (a) $e_{0} \in \operatorname{pcl}(O)$. Choose some history (b) $h_{0} \in H_{\langle O\rangle}$. We know by definition that $(c) p c l(O)<O$, hence $(d) e_{0}<O$, and we know from (a) that there is some history $h_{1}$ such that $(e) h_{1} \perp_{e_{0}} H_{\langle O\rangle}$. It follows from (b) and $(c)$ that $(f) \operatorname{pcl}(O) \subseteq h_{0}$, so that $\operatorname{pcl}(O)$ is consistent. Therefore $\operatorname{pcl}(O)-\left\{e_{0}\right\}$ can be partitioned as follows: $\mathbf{I}_{1}=\left\{e: e<e_{0}\right\} \cap p c l(O), \mathbf{I}_{2}=\left\{e: e_{0} \operatorname{SLR} e\right\} \cap$ $p c l(O)$, and $\mathbf{I}_{3}=\left\{e: e_{0}<e\right\} \cap p c l(O)$. $\mathbf{I}_{2} \subseteq h_{0}$, so that $\Pi_{\mathbf{I}_{2}}\left\langle h_{0}\right\rangle$ is defined. Since $\left\{e_{0}\right\}$ SLR $\mathbf{I}_{2}$, the absence of modal-correlation funny business (Assumption 4-2(2)) implies that there is a history $h_{1^{\prime}}$ such that $(g) h_{1^{\prime}} \in\left(\Pi_{e_{0}}\left\langle h_{1}\right\rangle \cap \Pi_{\mathbf{I}_{2}}\left\langle h_{0}\right\rangle\right.$ ). (If $\mathbf{I}_{2}=$ $\varnothing$, set $h_{1^{\prime}}=h_{1}$ and continue the argument.) By $(e)$ and $(g)$ (the left-hand part) and the transitivity of undividedness, (h) $h_{1^{\prime}} \perp_{e_{0}} H_{\langle O\rangle}$ so $h_{1^{\prime}} \notin H_{\langle O\rangle}$. For finishing the proof, it therefore suffices to show that $(z) h_{1^{\prime}} \in \bigcap_{e \in\left(p c l(O)-\left\{e_{0}\right\}\right)} H_{\left(e \nrightarrow \Pi_{e}\langle O\rangle\right)}$.

To this end, suppose that $(i) e \in\left(\operatorname{pcl}(O)-\left\{e_{0}\right\}\right)$, so that $(j) e<O$. For $(z)$ we need to show the conditional, if $(k) h_{1^{\prime}} \in H_{(e)}$ then $(y) h_{1^{\prime}} \in \Pi_{e}\langle O\rangle$, and so we suppose $(k)$. For $(y)$ we need only $h_{1^{\prime}} \equiv{ }_{e} H_{\langle O\rangle}$, and for this, by $(b)$ and $(j)$ and the transitivity of undividedness, we only need $(x) h_{1^{\prime}} \equiv_{e} h_{0}$. Step $(i)$ implies that $e$ belongs to one of $\mathbf{I}_{1}, \mathbf{I}_{2}$, and $\mathbf{I}_{3}$, and we argue by cases.

First, suppose that $e \in \mathbf{I}_{1}$, so that $(l) e<e_{0} . e_{0} \in\left(h_{0} \cap h_{1^{\prime}}\right)$ by $(b),(e)$ and $(g)$ (left-hand part), so $(x)$ by $(l)$.

Suppose next that $e \in \mathbf{I}_{2}$. Then $(x)$ comes at once from $(g)$ (the right-hand part). 
Last, suppose that $e \in \mathbf{I}_{3}$, i.e., $(m) e_{0}<e$ and $(n) e \in \operatorname{pcl}(O)$. This, however, cannot happen: $(k)$ and $(n)$ and $(f)$ imply that $e \in\left(h_{1^{\prime}} \cap h_{0}\right)$, so that with $(m)$ one would have $h_{1^{\prime}} \equiv_{e_{0}} h_{0}$, which contradicts $(h)$.

Theorem 5-3 is the "Hauptsatz" of this essay. (There are five more theorems to come, but each is a variation on Theorem 5-3.) I offer it as evidence that causae causantes in branching space-times constitute a truly interesting account of some aspects of causation when causation is taken to be "in the events."

\subsection{Inns conditions of scattered outcome events $\mathrm{O}$}

Outcome chains are not enough; we have five more candidates for what causes are causes of. A scattered outcome event is the next simplest case. Since s-o events are "conjunctive," occurring just in case all their parts occur, we shall again be looking for inns conditions rather than the inus conditions appropriate for disjunctive outcome events. Beyond that observation, one needs to be sure that the various aspects of the BST story about inns conditions for outcome chains properly adapt to inns conditions for s-o events. The modification for scattered outcome events $\mathbf{O}$ is to define the central concepts under the condition $e<{ }_{\exists} \mathbf{O}$. Since under that condition everything is strictly analogous to the case for $O$, I enter just the key definitions and one fact.

D23. $p c l(\mathbf{O})$, read "the set of prior causal loci of $\mathbf{O}, "={ }_{d f}\left\{e: e<{ }_{\exists} \mathbf{O} \& \exists h\left[h \perp_{e}\right.\right.$ $\left.\left.H_{\langle\mathbf{O}\rangle}\right]\right\}$.

When $e{ }_{\exists} \mathbf{O}, \Pi_{e}\langle\mathbf{O}\rangle$, read the projection of $\mathbf{O}$ onto $e,=_{d f}$ the unique member of $\Pi_{e}$ that is consistent with $H_{\langle\mathbf{O}\rangle}$. Alternatively and equivalently, $\Pi_{e}\langle\mathbf{O}\rangle{ }_{d f}\left\{h: h \equiv_{e} H_{\langle\mathbf{O}\rangle}\right\}$.

A causa causans or originating cause of $\mathbf{O}$ is defined as any basic primary transition $e \longmapsto \Pi_{e}\langle\mathbf{O}\rangle$ such that $e \in \operatorname{pcl}(\mathbf{O})$. In symbols:

$c c(\mathbf{O})$, read the set of causae causantes of $\mathbf{O},=_{d f}\left\{e \longmapsto \Pi_{e}\langle\mathbf{O}\rangle: e \in p c l(\mathbf{O})\right\}$.

Observe the following facts.

5-4 FACT. (About $\operatorname{pcl}(\mathbf{O})$ and $\left.\Pi_{e}\langle\mathbf{O}\rangle\right)$

1. " $e{ }_{\exists} \mathbf{O}$ ” in D23 is redundant (given no funny business).

2. $\operatorname{pcl}(\mathbf{O})=\bigcup o \in \mathbf{O} p c l(O)$.

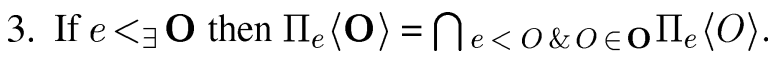

4. If $e<\exists \mathbf{O}$ then $H_{\left(e \nrightarrow \Pi_{e}\langle\mathbf{O}\rangle\right)}=\bigcap_{e<O \& O \in \mathbf{O}} H_{\left(e \nrightarrow \Pi_{e}\langle O\rangle\right)}$. 
Proof. Use Assumption 4-2(1) for an immediate proof of (1). The other facts fall out of the definitions.

The following theorem is in exact analogy to Theorem 5-3: It says that the causae causantes of a scattered outcome event $\mathbf{O}$ are inns conditions of $\mathbf{O}$.

\section{5-5 THEOREM. (Inns conditions of scattered outcome events $\mathbf{O}$ )}

1. Necessary condition. $H_{\langle\mathbf{O}\rangle} \subseteq \bigcap_{e \in p c l(\mathbf{O})} H_{\left(e \nrightarrow \Pi_{e}\langle\mathbf{O}\rangle\right)}$.

2. Jointly sufficient condition. $\bigcap_{e \in \operatorname{pcl}(\mathbf{O})} H_{\left(e \mapsto \Pi_{e}\langle\mathbf{O}\rangle\right)} \subseteq H_{\langle\mathbf{O}\rangle}$.

3. Non-redundancy. Suppose that $e_{0} \in \operatorname{pcl}(\mathbf{O})$. Then

$$
\bigcap_{e \in\left(p c l(\mathbf{O})-\left\{e_{0}\right\}\right)} H_{\left(e \hookrightarrow \Pi_{e}\langle\mathbf{O}\rangle\right)} \nsubseteq H_{\langle\mathbf{O}\rangle} .
$$

PROOF. Proofs of necessity, joint sufficiency, and non-redundancy are, given Fact 5-4, all corollaries of Theorem 5-3.

\subsection{Inus conditions for disjunctive outcome events $\breve{O}$}

As earlier indicated, a disjunctive outcome event $\breve{O}$ (a nonempty set of pairwiseinconsistent scattered outcome events) is to be interpreted disjunctively, so that the proper existence proposition is $H_{\langle\breve{\mathbf{O}}\rangle}={ }_{d f} \bigcup_{O \in \breve{\mathbf{O}}} H_{\langle O\rangle}$. That is, $H_{\langle\breve{\mathbf{O}}\rangle}$ occurs in a history $h$ iff some scattered outcome event in $\breve{\mathbf{O}}$ occurs in $h$. If "the birthday party" can be causally situated in more than one way, the disjunctive outcome event the birthday party is represented by a set of scattered outcome events, each of which represents a most-detailed causal situation in which the party takes place. For the first time we need the full Mackie idea of an inus condition, for we are distinguishing the various ways in which $\breve{O}$ might have been causally situated, which is to say, we are looking for a set of inns conditions for these situations, each of which is unnecessary (since $\breve{\mathbf{O}}$ might have occurred in virtue of another s-o event), but which taken together are jointly sufficient (and necessary).

A suitable definition of the cause-like loci for a disjunctive outcome event $\breve{O}$ is virtually forced:

D24. $\operatorname{pcl}(\breve{\mathbf{O}})$, read "the set of prior causal loci of $\breve{\mathbf{O}}, "={ }_{d f} \bigcup_{\mathbf{O} \in \breve{\mathbf{O}}} p c l(\mathbf{O})$.

A causa causans for a disjunctive outcome event $\breve{O}$ is defined as any causa causans for one of its members (its disjuncts).

$c c(\breve{\mathbf{O}})$, read "the set of causae causantes or originating causes of $\breve{\mathbf{O}}, "={ }_{d f}$ $\bigcup_{\mathbf{O} \in \breve{\mathbf{O}}^{c c}}(\mathbf{O})$. 
The idea is that each $H_{\left(e \hookleftarrow \Pi_{e}\langle\mathbf{O}\rangle\right)}$ for $e \in p c l(\breve{\mathbf{O}})$ and $\mathbf{O} \in \breve{\mathbf{O}}$ is an inus condition of $\breve{O}$ : an insufficient but non-redundant part of an unnecessary but sufficient condition of the occurrence of $\breve{O}$. That is,

5-6 THEOREM. (Inus conditions of disjunctive outcome events $\breve{\text { }) ~}$

$$
\bigcup_{\mathbf{O} \in \breve{\mathbf{O}}} \bigcap_{e \in p c l(\mathbf{O})} H_{\left(e \hookrightarrow \Pi_{e}\langle\mathbf{O}\rangle\right)}=H_{\langle\breve{\mathbf{O}}\rangle} .
$$

The outer union tells us that each element $\bigcap_{e \in \operatorname{pcl}(\mathbf{O})} H_{\left(e \mapsto \Pi_{e}\langle\mathbf{O}\rangle\right)}($ for $\mathbf{O} \in \breve{\mathbf{O}})$ is an unnecessary but sufficient condition of the occurrence of $\breve{\mathbf{O}}$. And the burden of Theorem 5-5 is precisely that the occurrence of each transition $e \longmapsto \Pi_{e}\langle\mathbf{O}\rangle$ (for $e$ $\in \operatorname{pcl}(\mathbf{O}))$ is an insufficient but non-redundant part of a sufficient (and necessary) condition of the occurrence of $\mathbf{O}$. Therefore, Theorem 5-6 says that the occurrence of each transition $e \longmapsto \Pi_{e}\langle\mathbf{O}\rangle$ (for $e \in \operatorname{pcl}(\breve{\mathbf{O}})$ ) is an inus condition for the occurrence of $\breve{O}$.

PROOF. Immediate from the definitions and Theorem 5-5.

There is one loose end. It might seem at first glance as if there is something imperfect if an inus condition for Oั is "used more than once," which could happen if $e \longmapsto \Pi_{e}\left\langle\mathbf{O}_{1}\right\rangle=e \longmapsto \Pi_{e}\left\langle\mathbf{O}_{2}\right\rangle$ for distinct $\mathbf{O}_{1}, \mathbf{O}_{2} \in \breve{\mathbf{O}}$. It needs but a moment's reflection, however, to appreciate that it is entirely normal for (even) mutually inconsistent s-o events to share some of their inns conditions.

\subsection{Inns and inus conditions of transition events}

"Absolute" explanations of outcome events live up to certain philosophical principles of completeness, but it is good to keep in mind a theme of Mackie 1974, which stresses that causation is typically to be relativized to a certain "field": The caused is not just an event, but an "event-in-a-certain-field," and furthermore "any part of the chosen field is decisively ruled out as a cause" (p. 35). Xu 1997 puts this idea in event form by means of his notion of a "preconditioning circumstance," an event that must "be established before the causing event has happened" (p. 162). $\mathrm{Xu}$ defines his notion of a preconditioning circumstance exactly, but all I use is the general idea, in part to adapt it to BST theory. What answers to a "preconditioning circumstance" must be an initial event $\mathbf{I}$ that is appropriately prior to the caused event (an outcome event $\bar{O}$ ). We may expect that $\mathbf{I}$ is a kind of "initial condition" that may play a causal role in the occurrence of $\bar{O}$. We have to allow in addition that some of the causes of the outcome $\bar{O}$ may occur properly after I (as in Xu 1997), and we furthermore have to realize that some may be spacelike related to the "conditioning circumstance" I, something that cannot happen in 


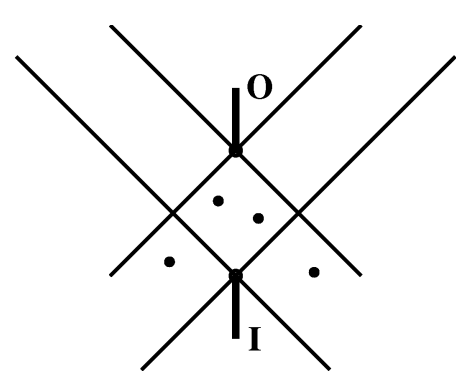

Figure 2: Some "boundary conditions"

Xu's framework of branching time. If we think of I as an "initial condition," then we may think of the causes that are either later or space-like related as "boundary conditions." Figure 2 shows a simple case, the unmarked dots representing point events that might count as loci for "boundary conditions."

We may think of this relativized case either as asking for causes of $\bar{O}$ "given $\mathbf{I}$," or, since we certainly require that $\mathbf{I}$ be appropriately prior to $\bar{O}$, we may ask for causes of the transition event $\mathbf{I} \longmapsto \bar{O}$. Without being clear on which alternative is preferable, I am going to follow up on the second. So we are asking for a causal account of a transition $\mathbf{I} \longmapsto O$ or $\mathbf{I} \longmapsto \mathbf{O}$ or $\mathbf{I} \longmapsto \breve{\mathbf{O}}$ from a given initial event to a certain outcome event. Since we are speaking of transition events, it must be presumed that the initial is appropriately prior to the outcome event (D9). We shall be looking for inns conditions of the transition events $\mathbf{I} \longmapsto O$ and $\mathbf{I} \longmapsto \mathbf{O}$, and inus conditions for transition events $\mathbf{I} \longmapsto \breve{\mathbf{O}}$. As before, the account of inus conditions comes swiftly from that for inns conditions, which it presupposes.

The key idea for inns conditions is that there is a "dropping off" of histories in the transition from $\mathbf{I}$ to $O[\mathbf{O}]$. Accordingly, in looking for a causal account of a transition event $\mathbf{I} \longmapsto O[\mathbf{I} \longmapsto \mathbf{O}]$, we wish to find splitting points only for those dropped histories, which is to say, for just those histories in $H_{[\mathbf{I}]}-H_{\langle O\rangle}$ $\left[H_{[\mathbf{I}]}-H_{\langle\mathbf{O}\rangle}\right]$. We entirely ignore those histories in which the initial event $\mathbf{I}$ does not finish. By so doing we may leave out of account some part of the total causal account of the outcome $\bar{O}$, but we will not be omitting anything relevant to the transition from $\mathbf{I}$ to $\bar{O}$. We are thereby led to the following definition of "past causal locus" for transition events.

D25. If $\mathbf{I} \longmapsto O$ is a transition event, then $\operatorname{pcl}(\mathbf{I} \longmapsto O)={ }_{d f}\left\{e: e<O \& \exists h\left[h \in H_{[\mathbf{I}]}\right.\right.$ $\left.\left.\& h \perp_{e} H_{\langle O\rangle}\right]\right\}$. 
If $\mathbf{I} \longmapsto \mathbf{O}$ is a transition event then $p c l(\mathbf{I} \longmapsto \mathbf{O})={ }_{d f}\left\{e: e<{ }_{\exists} \mathbf{O} \& \exists h\left[h \in H_{[\mathbf{I}]}\right.\right.$ $\left.\left.\& h \perp_{e} H_{\langle\mathbf{O}\rangle}\right]\right\}$.

If $\mathbf{I} \longmapsto \breve{\mathbf{O}}$ is a transition event, then $\operatorname{pcl}(\mathbf{I} \longmapsto \breve{\mathbf{O}})=_{d f} \bigcup_{\mathbf{O} \in \breve{\mathbf{O}}} p c l(\mathbf{I} \longmapsto \mathbf{O})$.

In each case, $p c l\left(\_\right)$should be read as "the set of past cause-like (or causal) loci of_."

Observe that if a certain point event $e$ is properly less than any member of $\mathbf{I}$, then such an $e$ cannot belong to $\operatorname{pcl}(\mathbf{I} \longmapsto O)$. So each member of $p c l(\mathbf{I} \longmapsto O)$ that is not itself a member of $\mathbf{I}$ must either be later than or space-like related to each member of $\mathbf{I}$. (To say that these are the loci of the "boundary conditions" of the transition gives a more accurate expression than the simpler account of "boundary conditions" given two paragraphs back.)

I still need to explain the idea of causa causans for transitions. Although it is by no means certain that it is most helpful, and even though it leads to a certain awkwardness, the suggestion is that we should not deviate from the idea of taking basic primary transitions $e \longmapsto \Pi_{e}\langle O\rangle$ as causae causantes.

D26. $c c(\mathbf{I} \longmapsto O)={ }_{d f}\left\{e \longmapsto \Pi_{e}\langle O\rangle: e \in \operatorname{pcl}(\mathbf{I} \longmapsto O)\right\}$.

$c c(\mathbf{I} \longmapsto \mathbf{O})=_{d f}\left\{e \longmapsto \Pi_{e}\langle\mathbf{O}\rangle: e \in p c l(\mathbf{I} \longmapsto \mathbf{O})\right\}$.

$c c(\mathbf{I} \longmapsto \breve{\mathbf{O}})={ }_{d f} \bigcup_{\mathbf{O} \in \breve{\mathbf{O}}} c c(\mathbf{I} \longmapsto \mathbf{O})$.

In each case, $c c\left(\_\right)$should be read as "the set of causae causantes (or originating causes) of _."

As verification, the following should turn out. Then we are told exactly what had to happen to account for the transition from $\mathbf{I}$ to $O$.

5-7 THEOREM. (Inns conditions of $\mathbf{I} \longmapsto O$ ) Assume that $\mathbf{I} \longmapsto O$ is a transition event.

1. Necessary condition. $\left.H_{[\mathbf{I}]} \cap H_{(\mathbf{I} \mapsto O)} \subseteq \bigcap_{e \in p c l(\mathbf{I} \mapsto O)} H_{\left(e \hookrightarrow \Pi_{e}\langle O\rangle\right)}\right)$.

2. Jointly sufficient condition. $\bigcap_{e \in p c l(\mathbf{I} \mapsto O)} H_{\left(e \mapsto \Pi_{e}\langle O\rangle\right)} \subseteq H_{(\mathbf{I} \mapsto O)}$

3. Non-redundancy. Suppose that $e_{0} \in \operatorname{pcl}(\mathbf{I} \longmapsto O)$. Then

$$
\bigcap_{e \in\left(p c l(\mathbf{I} \hookrightarrow O)-\left\{e_{0}\right\}\right)} H_{\left(e \hookrightarrow \Pi_{e}\langle O\rangle\right)} \nsubseteq H_{(\mathbf{I} \hookrightarrow O)} .
$$

The awkwardness I mentioned is seen in the part of the theorem stating "necessary condition." It would be more attractive (but false) to say that each causa causans is a necessary condition of just the transition $\mathbf{I} \longmapsto O$. Perhaps another way of looking at the matter is more elegant. 
PROOF. Necessity and joint sufficiency are unproblematic. The proof of nonredundancy needs to be adjusted. Aside from changing " $p c l(O)$ " to " $p c l(\mathbf{I} \longmapsto O)$," the chief adjustment is that at step (e) (in a proof of this theorem that corresponds to that of Theorem 5-3) we know in addition that $\left(e^{\prime}\right) h \in H_{[\mathbf{I}]}$, and the second adjustment is that at the step corresponding to $(f)$ we know that $\left(f^{\prime}\right)(\operatorname{pcl}(\mathbf{I} \longmapsto O)$ $\cup \mathbf{I}) \subseteq h_{0}$. We may then redefine $\mathbf{I}_{2}$ as $\left\{e: e_{0} \operatorname{SLR} e\right\} \cap(p c l(\mathbf{I} \longmapsto O) \cup \mathbf{I})$. Then the argument goes along as before, except that at $(z)$ we are not yet done; we still must verify that $h_{1^{\prime}} \in H_{[\mathbf{I}]}$ in order to be sure that $h_{1^{\prime}} \notin H_{(\mathbf{I} \rightarrow O)}$. Choose $e \in \mathbf{I}$; we need to show that $e \in h_{1^{\prime}}$. Since $e$ is consistent with $e_{0}$ (both belong to $h_{0}$ and indeed to $h_{1}$ as well), there are four cases. $e_{0}<e$ is impossible: Since $e \in\left(h_{0} \cap h_{1}\right)$, that would conflict with (e). If $e<e_{0}$ or if $e=e_{0}$, we may be sure that $e \in h_{1^{\prime}}$ since by ( $g$ ) (left-hand part) $e_{0} \in h_{1^{\prime}}$. And if $e_{0} \operatorname{SLR} e$, then the right-hand part of ( $g$ ) (with the definition of $\mathbf{I}_{2}$ modified as indicated) implies that $e \in h_{1^{\prime}}$; which completes the argument for non-redundancy.

One might think to generalize: Instead of an initial event $\mathbf{I}$, one might have only a set of histories $H$, a proposition, a "fact." One is asking for "Why did $O$ occur, given $H$ ?" For this to make sense, you presumably require that $H_{\langle O\rangle} \subseteq H$, and you are asking for why $O$ occurred instead of some alternative in $H$. Since $H$ is "given," you are not asking about why $H$ instead of something else. The question makes perfect sense, and its structure deserves investigation. In this case, however, the present account by no means guarantees that you will find an inns condition. That the "given" is the occurrence of a prior initial event $\mathbf{I}$ appears to be essential for the present result; or at least it would seem so from the proof of Theorem 5-7.

As before, I extend to transition events the outcomes of which are scattered; proof is omitted.

5-8 THEOREM. (Inns conditions of $\mathbf{I} \longmapsto \mathbf{O}$ ) Assume that $\mathbf{I} \longmapsto \mathbf{O}$ is a transition event.

1. Necessary condition. $\left.H_{[\mathbf{I}]} \cap H_{(\mathbf{I} \mapsto \mathbf{O})} \subseteq \bigcap_{e \in p c l(\mathbf{I} \longmapsto \mathbf{O})} H_{\left(e \mapsto \Pi_{e}\langle\mathbf{O}\rangle\right)}\right)$.

2. Jointly sufficient condition. $\bigcap_{e \in p c l(\mathbf{I} \mapsto \mathbf{O})} H_{\left(e \nrightarrow \Pi_{e}\langle\mathbf{O}\rangle\right)} \subseteq H_{(\mathbf{I} \hookrightarrow \mathbf{O})}$

3. Non-redundancy. Suppose that $e_{0} \in \operatorname{pcl}(\mathbf{I} \longmapsto \mathbf{O})$. Then

$$
\bigcap_{e \in\left(p c l(\mathbf{I} \hookrightarrow \mathbf{O})-\left\{e_{0}\right\}\right)} H_{\left(e \hookrightarrow \Pi_{e}\langle\mathbf{O}\rangle\right)} \nsubseteq H_{(\mathbf{I} \succ \mathbf{O})} .
$$

A question of some interest because confusing is this: What if the "effect" transition event is itself a basic primary transition event $e_{0} \longmapsto \Omega_{e_{0}}\left\langle h_{0}\right\rangle$ (D13, D14)? What are its causae causantes? You can calculate as a technical matter that $p c l\left(e_{0}\right.$ $\left.\longmapsto \Omega_{e_{0}}\left\langle h_{0}\right\rangle\right)=\left\{e_{0}\right\}$, noting that $e_{0}$ is in the past of the outcome of the "effect" 
transition $e_{0} \longmapsto \Omega_{e_{0}}\left\langle h_{0}\right\rangle$. Therefore, $c c\left(e_{0} \longmapsto \Omega_{e_{0}}\left\langle h_{0}\right\rangle\right)=\left\{e_{0} \longmapsto \Pi_{e_{0}}\left\langle h_{0}\right\rangle\right\}$. Since, however, by Fact 4-1 $\Pi_{e_{0}}\left\langle h_{0}\right\rangle$ is nothing but the propositional surrogate of the scattered outcome event $\Omega_{e_{0}}\left\langle h_{0}\right\rangle$, the fact is that $e_{0} \longmapsto \Omega_{e_{0}}\left\langle h_{0}\right\rangle$ is its own causa causans. Call this "self-causation" or not just as you prefer. In any case, the technical fact corresponds to the conceptual point that your why-questions must come to an end when you reach a causa causans. There can be non-trivial causal reasons for the occurrence of the initial event $e_{0}$, and also of the occurrence of the scattered outcome event $\Omega_{e_{0}}\left\langle h_{0}\right\rangle$, but for the transition event $e_{0} \longmapsto \Omega_{e_{0}}\left\langle h_{0}\right\rangle$ there is nothing more to say. Warning: Do not metaphor this situation into obfuscation with phrases such as "pops up from nowhere" (Mackie, p. 177). ${ }^{19}$

What about transitions to disjunctive outcome events? As before, the idea is that once we have inns conditions for transitions to scattered outcome events, we automatically have inus conditions for transitions to disjunctive outcome events. Proof is omitted.

5-9 THEOREM. (Inus conditions of $\mathbf{I} \longrightarrow \breve{\mathbf{O}}$ ) Assume that $\mathbf{I} \longmapsto \breve{\mathbf{O}}$ is a transition event.

$$
\begin{aligned}
& \bigcup_{\mathbf{O} \in \breve{\mathbf{O}}} \bigcap_{e \in p c l(\mathbf{I} \succ \mathbf{O})} H_{\left(e \hookleftarrow \Pi_{e}\langle\mathbf{O}\rangle\right)} \subseteq H_{(\mathbf{I} \hookleftarrow \breve{\mathbf{O}})}, \text { and (awkwardly) } \\
& H_{[\mathbf{I}]} \cap H_{(\mathbf{I} \hookrightarrow \breve{\mathbf{O}})} \subseteq \bigcup_{\mathbf{O} \in \breve{\mathbf{O}}} \bigcap_{e \in p c l(\mathbf{I} \hookrightarrow \mathbf{O})} H_{\left(e \hookleftarrow \Pi_{e}\langle\mathbf{O}\rangle\right)}
\end{aligned}
$$

\section{Counterfactual conditionals}

What about counterfactual conditionals? One might wish a Stalnaker-Lewis account based on "similarity." Müller 2002 and Placek 2002a have considered this matter, with suggestive but inconclusive results. Müller and Placek exploit the intuition that comparative similarity between histories can be made to depend on splitting later rather than earlier, and idea also present in Xu's branching-time framework. The inconclusiveness arises, as I see it, from the fact that the causal language of branching space-times is, as it stands, not sufficiently rich to ground similarity-statements in any but simple cases.

Here is one more try, appealing to the apparatus of causae causantes. Position yourself at some $e_{1}$ after some portion of an outcome chain $O$, so that $H_{\left(e_{1}\right)}$ is the set of (indexically) "actual" histories, among which you will find all histories in which $O$ occurs. You can well say of an inns condition $e \longmapsto \Pi_{e}\langle O\rangle$ for the occurrence of $O$ that if it had not occurred then $O$ would not have occurred. This is just a strict conditional. It is possible, however, to go a little further. I suppose a

\footnotetext{
${ }^{19}$ Mackie in this passage is discussing the idea of "causal chains," so that this is a good place to observe that the idea of a causa causans in BST theory does not depend on some such notion.
} 
history $h$ in which "all the other causae causantes of $O$ occur but the one does not" is very "close" to those in $H_{\left(e_{1}\right)}$, which perhaps would at least loosely connect my use of the subjunctive conditional with the theories of Stalnaker and Lewis. Indeed, in the imagined circumstance one is entitled to a somewhat more informative strict conditional: If $e \longmapsto \Pi_{e}\langle O\rangle$ had not occurred then what would have happened at $e$ is some alternative to $\Pi_{e}\langle O\rangle$. Consider that slowly: Because of the "material implication" sense of the occurrence of $e \longmapsto \Pi_{e}\langle O\rangle$, to say that it did not or would not occur is to say both that $e$ did or would occur, and that $\Pi_{e}\langle O\rangle$ did not or would not occur. Hence, some other alternative $\Pi_{e}\langle h\rangle$ in $\Pi_{e}$ would have occurred. Got it?

Still, the fundamental point is that the idea of non-redundancy is expressed by holding fixed all of the other originating causes of the outcome event $O$ in question, permitting only the one causa causans to fail; and that seems to give some taste of "similarity" or "closeness." Thus, if by the counterfactual "if $e \longmapsto \Pi_{e}\langle O\rangle$ had not occurred then ..." you systematically mean the strict conditional "if $e \longmapsto \Pi_{e}\langle O\rangle$ had not occurred but all the other causae causantes of $O$ occurred, then ...," nonredundancy guarantees that you will make sense by having said something with a consistent antecedent, and something that is perhaps in the spirit of Stalnaker and Lewis. A treatment such as this would, given certain idealizing assumptions, apply to examples such as "if Fred had not bet on tails he would have won" even though Fred's winning also depends on how the coin comes up.

What really seems best to me, however, is to abandon the belief that there is a widely useful objective theory of counterfactuals based on similarity. The Stalnaker-Lewis counterfactuals are helpful in analyzing conversations, but, in my view, not useful in framing objective theories of e.g. causation. They are insufficiently robust. In place of Stalnaker-Lewis counterfactuals, BST theory offers "strict" conditionals based on universally quantifying over histories such as the following (assuming that the bet is placed in the causal past of the flip): "If the coin had come up heads, then regardless of how history worked itself out from that point forward, you would have won your bet." But the following would be false as an objective strict conditional and thus, in spite of its undoubted appeal to our feelings, would have no objective merit: "If you had bet on heads, then regardless of how history worked itself out from that point forward, you would have won your bet." 20

\footnotetext{
${ }^{20}$ R. K. Meyer has suggested that counterfactuals should be left to sportscasters and military historians.
} 


\section{Appendix: Tense and modal connectives in BST}

It may heighten understanding to consider an appropriate language for speakers inhabiting Our World. This discussion, which in several places substantially repeats parts of $\S \mathbf{3}$ of Müller 2002, follows chapter 8 of Belnap et al. 2001 as closely as possible. I suppose that truth and denotation are parameterized with a model $\mathcal{M}$ $=\langle\mathcal{S}, \mathcal{J}\rangle$, where $\mathcal{S}$ is a BST structure (of which Our World ordered by $<$ is the paradigm) and $\mathcal{J}$ is an interpretation-function giving appropriate meaning to each "non-logical" constant of the language. In addition, there is a context parameter, $e_{c}$, a point event that represents the (idealized) standpoint of an utterance, and two auxiliary parameters, the point event of evaluation $e$ and the history of evaluation $h$, which I always write as $e / h$ in order to highlight the requirement that $e \in h$. I write

$$
\mathcal{M}, e_{c}, e / h \vDash A
$$

to express that sentence $A$ is true with respect to model $\mathcal{M}$, context point event $e_{c}$, point event of evaluation $e$, and history of evaluation $h$.

\section{7-1 DeFinition. (Tense-modal connectives in BST)}

1. Future tense in BST. $\mathcal{M}, e_{c}, e / h \vDash$ Will: $A \leftrightarrow_{d f} \exists e_{1}\left[e<e_{1}\right.$ and $e_{1} \in h$ and $\left.\mathcal{M}, e_{c}, e_{1} / h \vDash A\right]$. READing: It will be true that $A$. (The "will" refers to the Minkowski-like causal future in $h$, not to the temporal future relative to some frame of reference as contemplated in note 7 of Müller 2002.)

2. Past tense in BST. $\mathcal{M}, e_{c}, e / h \vDash$ Was: $A \leftrightarrow_{d f} \exists e_{1}\left[e_{1}<e\right.$ and $e_{1} \in h$ and $\mathcal{M}$, $\left.e_{c}, e_{1} / h \vDash A\right]$. READING: It was true that $A$. (The "was" refers to the causal past, not to the temporal past relative to some frame of reference.)

3. $\mathcal{M}, e_{c}, e / h \vDash A t_{t}: A$ iff the denotation of the singular term $t$ at $\left\langle\mathcal{M}, e_{c}, e / h\right\rangle$ is a point event $e_{1}$ such that $e_{1} \in h$ and $\mathcal{M}, e_{c}, e_{1} / h \vDash A$. READING: At $t, A$.

4. Settledness in BST. $\mathcal{M}, e_{c}, e / h \vDash S e t t: A \leftrightarrow_{d f} \forall h_{1}\left[e \in h_{1} \rightarrow \mathcal{M}, e_{c}, e / h_{1} \vDash\right.$ $A]$. READING: It is settled true that $A$.

It is good to adapt the idea of settledness to propositions in BST theory: $H$ is settled true at $e \leftrightarrow_{d f} H_{(e)} \subseteq H$.

5. Historical possibility in BST is the dual of settledness. $\mathcal{M}, e_{c}, e / h \vDash$ Poss: $A$ $\leftrightarrow_{d f} \exists h_{1}\left[e \in h_{1}\right.$ and $\left.\mathcal{M}, e_{c}, e / h_{1} \vDash A\right]$. READING: It is (historically) possible that $A$. 
6. Actuality/factuality in BST. $\mathcal{M}, e_{c}, e / h \vDash A c t u a l l y: A \leftrightarrow_{d f} \forall h_{1}\left[e_{c} \in h_{1} \rightarrow \mathcal{M}\right.$, $\left.e_{c}, e_{c} / h_{1} \vDash A\right]$.

7. Happening in BST. $\mathcal{M}, e_{c}, e / h \vDash H a p p: A \leftrightarrow_{d f}$ both of the following.

- Positive condition. $\forall h_{1}\left[h_{1} \equiv_{e} h \rightarrow \mathcal{M}, e_{c}, e / h_{1} \vDash A\right]$.

- Negative condition. $\exists h_{1}\left[e \in h_{1}\right.$ and $\left.\mathcal{M}, e_{c}, e / h_{1} \not \models A\right]$.

READING: It so happens that $A .^{21}$

8. It so happens that it remains possible that $A \leftrightarrow_{d f}$ Happ:Will:Poss: $A$.

9. Same thing semi-metalinguistically: At $e$ on $h$ it so happens that it remains possible that $A \leftrightarrow_{d f} \mathcal{M}, e_{c}$, elh $\vDash$ Happ:Will:Poss: $A$.

10. Same thing purely metalinguistically: $:^{22}$ At $e$ on $h$ it so happens that $A$ remains possible $\leftrightarrow_{d f} \mathcal{M}, e_{c}$, elh $\vDash$ Happ:Will:Poss: $A$.

11. The underlying ideas may also be adapted to make sense for "point-eventdependent propositions," which are sets of point-event-histories pairs $e / h$ such that $e \in h$. Let $H^{+}$range over point-event-dependent propositions (sets of $e / h$ pairs such that $e \in h$ ). The connectives defined above are turned into operations on such propositions by fixing model $\mathcal{N}$ and context $e_{c}$, and just replacing " $\mathcal{M}, e_{c}, e / h \vDash A$ " by " $e / h \in H^{+}$."

12. In order to interface point-event-dependent propositions $H^{+}$with propositions $H$ as sets of histories, I introduce a couple of operations that go between the two notions. Where $H$ is a set-of-histories proposition, $E^{+}: H$ gives the set of all pairs $e / h$ such that $e \in h$ and $h \in H$. Where $H^{+}$is setof-point-event-history-pairs proposition, $E^{-}: H^{+}$gives the set of histories $h$ such that $e / h \in H^{+}$for some $e \in h$. Evidently $E^{-}: E^{+}: H=H$.

13. With all this baggage, we can say the following about the construct $\Pi_{e}\langle O\rangle$ of D18.

$$
\Pi_{e}\langle O\rangle=E^{-}: A t_{e}: \text { Happ:Will:Poss: } E^{+}: H_{\langle O\rangle} .
$$

\footnotetext{
${ }^{21}$ Happening is defined in a closely related way in Xu 1997. There is an inevitable artificiality about our reading here. The semantics is patterned after the dstit of Belnap et al. 2001, but without an agent. Note that the present tense of "happens," like that of dstit, is history-dependent, but the past tense is not (if it happened, then it is settled that it happened, i.e., if Was:Happ:A then Sett:Was:Happ:A).

${ }^{22}$ Here " $A$ " is used in term position, whereas in (9), " $A$ " is used once in sentential position and once in term position.
} 


\section{References}

Belnap, N. 1992, "Branching space-time”, Synthese 92, 385-434.

Belnap, N. 1999, "Concrete transitions", in G. Meggle, ed., Actions, norms, values: Discussions with Georg Henrik von Wright, Walter de Gruyter, Berlin, pp. 227-236. A "postprint" (2002) may be obtained from http://philsci-archive.pitt.edu.

Belnap, N. 2002a, "EPR-like 'funny business' in the theory of branching spacetimes", in Placek and Butterfield 2002, pp. 293-315. A preprint of this essay may be obtained from http://philsci-archive.pitt.edu.

Belnap, N. 2002b, No-common-cause EPR-like funny business in branching space-times. A preprint of this essay may be obtained from http:// philsci-archive.pitt.edu.

Belnap, N., Perloff, M. and Xu, M. 2001, Facing the future: Agents and choices in our indeterminist world, Oxford University Press, Oxford.

Mackie, J. L. 1974, The cement of the universe, Oxford University Press, Oxford.

Müller, T. 2002, "Branching space-time, modal logic and the counterfactual conditional", in Placek and Butterfield 2002, pp. 273-291.

Placek, T. 2002a, Comparative similarity in branching space-times. To appear in International Journal for Theoretical Physics.

Placek, T. 2002b, "Partial indeterminism is enough: a branching analysis of Belltype inequalities", in Placek and Butterfield 2002, pp. 317-342.

Placek, T. and Butterfield, J., eds 2002, Non-locality and modality, Kluwer Academic Publishers, Dordrecht.

Szabo, L. and Belnap, N. 1996, "Branching space-time analysis of the GHZ theorem", Foundations of physics 26(8), 989-1002.

Vendler, Z. 1962, "Effects, results, and consequences", in R. J. Butler, ed., Analytical philosophy, 1st series, Oxford ??? ???, Oxford, pp. ??-??

von Kutschera, F. 1986, “Bewirken”, Erkenntnis 24, 253-281.

von Kutschera, F. 1993, “Causation”, Journal of philosophical logic 22, 563-588. 
von Wright, G. H. 1963, Norm and action. A logical inquiry, Routledge and Kegan Paul, London.

Wölfl, S. 2002, Events in treelike structures. Unpublished preprint.

Xu, M. 1997, "Causation in branching time (I): transitions, events and causes", Synthese 112, 137-192. 


\section{Contents}

1 Introduction 1

2 The cement of the universe 3

3 Preliminaries 5

3.1 First definitions and postulates ............. 5

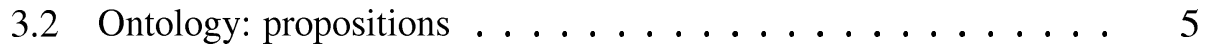

3.3 Ontology: initial events . . . . . . . . . . . 7

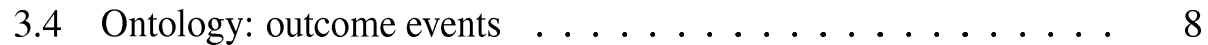

3.5 Ontology: transition events . . . . . . . . . . . . . 11

3.6 Propositional language applied to events . . . . . . . . . . 12

4 Causae causantes $\quad 13$

4.1 Causae causantes are basic primary transition events . . . . . . 13

4.2 Causae causantes of an outcome chain . . . . . . . . . . 16

4.3 No funny business . . . . . . . . . . . . . . . . 18

5 Causae causantes and inns and inus conditions 20

5.1 Inns conditions of outcome chains $O$ : not quite $\ldots \ldots \ldots \ldots 22$

5.2 Inns conditions of outcome chains $O \ldots \ldots \ldots \ldots$

5.3 Inns conditions of scattered outcome events $\mathbf{O} \ldots \ldots \ldots 25$

5.4 Inus conditions for disjunctive outcome events $\breve{\mathbf{O}} \ldots \ldots \ldots$

5.5 Inns and inus conditions of transition events . . . . . . . 27

6 Counterfactual conditionals 31

7 Appendix: Tense and modal connectives in BST 33 